\title{
Functional ARCH and GARCH Models: A Yule-Walker Approach
}

\author{
Sebastian Kühnert*
}

December 15, 2019

\begin{abstract}
Conditional heteroskedastic financial time series are commonly modelled by ARCH and GARCH. $\mathrm{ARCH}(1)$ and GARCH processes were recently extended to the function spaces $C[0,1]$ and $L^{2}[0,1]$, their probabilistic features were studied and their parameters were estimated. The projections of the operators on a finite-dimensional subspace were estimated, as were the complete operators in $\operatorname{GARCH}(1,1)$. An explicit asymptotic upper bound for the estimation errors was stated in $\mathrm{ARCH}(1)$. This article provides sufficient conditions for the existence of strictly stationary solutions, weak dependence and finite moments of ARCH and GARCH processes in various $L^{p}[0,1]$ spaces, $C[0,1]$ and other spaces. In $L^{2}[0,1]$ we deduce explicit asymptotic upper bounds of the estimation errors for the shift term and the complete operators in $\mathrm{ARCH}$ and GARCH and for the projections of the operators on a finite-dimensional subspace in ARCH. The operator estimaton is based on Yule-Walker equations. The estimation of the GARCH operators also involves a result concerning the estimation of the operators in invertible, linear processes which is valid beyond the scope of $\mathrm{ARCH}$ and GARCH. Through minor modifications, all results in this article regarding functional $\mathrm{ARCH}$ and $\mathrm{GARCH}$ can be transferred to functional ARMA.
\end{abstract}

Keywords: ARCH and GARCH processes; ARMA processes; functional data; invertible linear processes; parameter estimation; stationary solutions; Yule-Walker equations

AMS 2010 subject classifications: 47B38, 60G10, 62F12

\section{Introduction}

Volatility, usually measured by the variance, is one of the essential objects of study of financial time series. These are often strictly stationary but conditional heteroskedastic, where latter means that the variances at any time conditioned on the past are non-constant and randomly changing. A popular model exhibiting this phenomenon is the autoregressive conditional heteroskedasticity (ARCH) model established by Engle (1982), for which he was awarded the noble prize in economics in 2003. This model was extended to the generalized ARCH (GARCH) model by Bollerslev (1986). Various authors established modifications of univariate and multivariate ARCH and GARCH processes, studied their probabilistic properties and estimated their parameters. The books Andersen et al. (2009), Francq \& Zakoïan (2010) and Gouriéroux (1997) provide an excellent overview and applications of such processes. Due to a progress in processing techniques and since high-resolution tick data are accessible and can be described as functions, it seems reasonable to extend these models on infinite-dimensional spaces, enabling the analysis to be more accurate. Such an extension, from a mathematical point of view, is unproblematic for complete, separable metric spaces $M$ since completeness of $M$ implies that the Borel $\sigma$-field $\mathbb{B}(M)$ is well defined and separability ensures that e.g. sums of random variables are random variables again (see Ledoux \& Talagrand (1991)) A detailed introduction in Functional Data and Functional Time Series Analysis, the areas dealing with random variables resp. time series with values in an infinite-dimensional space (mostly spaces of functions with domain $[0,1]^{d}$ with $d \in \mathbb{N}$ ), can be found in Bosq (2000), Ferraty \& Vieu (2006), Hsing \& Eubank (2015) and Ramsay \& Silverman (1997), and for a compact synopsis (in German), see Kühnert (2019).

\footnotetext{
*Institute of Mathematics, University of Rostock, D-18051 Rostock, Germany, EMAIL: s.kuehnert_math@gmx.de
} 
Hörmann et al. (2013) made the initial step by introducing $\mathrm{ARCH}(1)$ processes with values in the spaces $C[0,1]$ and $L^{2}[0,1]$ of continuous resp. of square-integrable real valued functions with domain $[0,1]$. They established sufficient conditions for the existence of strictly stationary solutions, finite moments and weak dependence. In $L^{2}[0,1]$ they constructed consistent estimators and stated explicit asymptotic upper bounds of the estimation errors for the shift term and the projections of the operator on finite-dimensional subspaces by assuming the operator to be an integral operator and estimating its kernel. Aue et al. (2017) established $\operatorname{GARCH}(1,1)$ processes in $C[0,1]$ and in $L^{2}[0,1]$ and found sufficient conditions for the existence of strictly stationary solutions, finite moments and weak dependence. In $L^{2}[0,1]$ they derived a consistent least squares estimator for the projections of the parameters on finite-dimensional subspaces but without stating an explicit asymptotic upper bound of the estimation errors. At last, Cerovecki et al. (2019) studied $L^{2}[0,1]$-valued $\operatorname{GARCH}(\mathfrak{p}, \mathfrak{q})$ processes for integers $\mathfrak{p}, \mathfrak{q}>0$. They developed sufficient conditions for the existence of strictly stationary solutions and finite moments. By a quasi-likelihood approach, the projections of the parameters on a finite-dimensional subspace and only for $\mathfrak{p}=1=\mathfrak{q}$ the complete operators were estimated consistently. In both cases, no explicit asymptotic upper bound of the estimation errors was stated. Hörmann et al. (2013), Aue et al. (2017) and Cerovecki et al. (2019) also provided simulation studies showing how their models matched with real data and illustrated possible applications. For further work dealing with functional ARCH and GARCH models, see Kokoszka et al. (2017) and Rice et al. (2019). Moreover, for the estimation procedure of operators in general Banach spaces, see Ruiz-Medina \& Álvarez-Liébana (2019).

In this article, we establish $\operatorname{ARCH}(\mathfrak{p})$ and $\operatorname{GARCH}(\mathfrak{p}, \mathfrak{q})$ processes for all $\mathfrak{p}, \mathfrak{q} \in \mathbb{N}$ with values in $L^{p}[0,1]$ with $p \in[1, \infty), C[0,1]$ and other spaces. We provide sufficient conditions for the existence of strictly stationary solutions, weak dependence and moments of these time series under mild conditions. The focus of this paper is on deducing estimators for the shift term and the complete operators of $L^{2}[0,1]$-valued $\operatorname{ARCH}(\mathfrak{p})$ and $\operatorname{GARCH}(\mathfrak{p}, \mathfrak{q})$ processes for any $\mathfrak{p}, \mathfrak{q} \in \mathbb{N}$ and on deriving explicit asymptotic upper bounds of their estimation errors. We also deduce explicit asymptotic upper bounds of the estimation errors for the operators on a finite-dimensional subspace of these ARCH processes. The operator estimation in this work is always based on Yule-Walker equations and the estimators for the GARCH operators also involve estimators for the operators of invertible, linear processes represented as inverted time series. We derive explicit asymptotic upper bounds for the estimation errors of these estimators. Also, this upper bound holds for the estimation errors when estimating the operators in the associated linear process and is valid beyond the context of functional ARCH and GARCH models. All results in this article regarding functional ARCH and GARCH can be transferred to functional ARMA processes due to their relationship.

In this paper, we use the following notation. $a \wedge b:=\min (a, b)$ and $a \vee b:=\max (a, b)$ for $a, b \in \mathbb{R}$. For functions $f, g: \mathbb{D} \subseteq \mathbb{R} \rightarrow \mathbb{R}$, we write $f \propto g$ resp. $f \precsim g$ if there is a $c \in \mathbb{R}$ with $f(x)=c g(x)$ resp. $f(x) \leq c g(x)$ for all $x \in \mathbb{D}$. For sequences $\left(a_{n}\right)_{n \in \mathbb{N}},\left(b_{n}\right)_{n \in \mathbb{N}} \subseteq(0, \infty)$, we write $a_{n} \sim b_{n}$ if $\frac{a_{n}}{b_{n}} \rightarrow 1, a_{n} \asymp b_{n}$ if $a_{n} \sim c b_{n}$ for some $c \neq 0, a_{n}=\omega\left(b_{n}\right)$ if $b_{n}=\mathrm{o}\left(a_{n}\right)$ (for $\left.n \rightarrow \infty\right)$ and $a_{n}=\Omega\left(b_{n}\right)$ if $b_{n}=\mathrm{O}\left(a_{n}\right)$ (for $n \rightarrow \infty$ ). Further, $\Xi\left(a_{n}, b_{n}\right):=\omega\left(a_{n}\right) \cap \mathrm{o}\left(b_{n}\right), \Xi\left[a_{n}, b_{n}\right):=\Omega\left(a_{n}\right) \cap \mathrm{o}\left(b_{n}\right), \Xi\left(a_{n}, b_{n}\right]:=\omega\left(a_{n}\right) \cap \mathrm{O}\left(b_{n}\right)$ and $\Xi\left[a_{n}, b_{n}\right]:=\Omega\left(a_{n}\right) \cap \mathrm{O}\left(b_{n}\right)$. By $0_{V}$ we denote the identity element of addition of a vector space $V$ and $V^{n}:=\left\{\left(v_{1}, \ldots, v_{n}\right)^{T} \mid v_{1}, \ldots, v_{n} \in V\right\}$, with $n \in \mathbb{N}$, becomes a vector space by our componentwise definition of scalar multiplication and vector addition. For a space $F$ of functions $f:[0,1] \rightarrow \mathbb{R}, F_{>0}$ and $F_{\geq 0}$ denote the sets of functions $f \in F$ with $f(t)>0$ resp. $f(t) \geq 0$ for $\lambda$-a.e. $t \in[0,1]$ where $\lambda$ is the Lebesgue-Borel measure on $[0,1]$, and $f \odot g$ denotes the pointwise product of $f, g \in F$ if it is well-defined. Let $\left(\mathcal{B},\|\cdot\|_{\mathcal{B}}\right),\left(\mathcal{B}^{\prime},\|\cdot\|_{\mathcal{B}^{\prime}}\right)$ be Banach spaces and $\left(\mathcal{H},\langle\cdot, \cdot\rangle_{\mathcal{H}}\right),\left(\mathcal{H}^{\prime},\langle\cdot, \cdot\rangle_{\mathcal{H}^{\prime}}\right)$ be Hilbert spaces. On Hilbert spaces we always use norms induced by their scalarproduct and we say CONS for a complete orthonormal system. We endow Banach spaces $\left(\mathcal{B}^{n},\|\cdot\|_{\mathcal{B}^{n}}\right)$ with the norm $\|\boldsymbol{b}\|_{\mathcal{B}^{n}}^{2}:=\sum_{i=1}^{n}\left\|b_{i}\right\|_{\mathcal{B}}^{2}$ where $\boldsymbol{b}:=\left(b_{1}, \ldots, b_{n}\right)^{T} \in \mathcal{B}^{n}$ and Hilbert spaces $\left(\mathcal{H}^{n},\langle\cdot, \cdot\rangle_{\mathcal{H}^{n}}\right)$ with the scalarproduct $\langle\boldsymbol{h}, \tilde{\boldsymbol{h}}\rangle_{\mathcal{H}^{n}}:=\sum_{i=1}^{n}\left\langle h_{i}, \tilde{h}_{i}\right\rangle_{\mathcal{H}}$ where $\boldsymbol{h}:=\left(h_{1}, \ldots, h_{n}\right)^{T}, \tilde{\boldsymbol{h}}:=\left(\tilde{h}_{1}, \ldots, \tilde{h}_{n}\right)^{T} \in \mathcal{H}^{n}$. We write $\mathcal{L}_{\mathcal{B}, \mathcal{B}^{\prime}}, \mathcal{K}_{\mathcal{B}, \mathcal{B}^{\prime}}, \mathcal{S}_{\mathcal{B}, \mathcal{B}^{\prime}}$ resp. $\mathcal{N}_{\mathcal{B}, \mathcal{B}^{\prime}}$ for the space of bounded, compact, Hilbert-Schmidt resp. nuclear operators from $\mathcal{B}$ to $\mathcal{B}^{\prime}$ with $\mathcal{L}_{\mathcal{B}}=\mathcal{L}_{\mathcal{B}, \mathcal{B}}, \mathcal{K}_{\mathcal{B}}=\mathcal{K}_{\mathcal{B}, \mathcal{B}}, \mathcal{S}_{\mathcal{B}}=\mathcal{S}_{\mathcal{B}, \mathcal{B}}$ and $\mathcal{N}_{\mathcal{B}}=\mathcal{N}_{\mathcal{B}, \mathcal{B}}$ where the term operator always refers to a linear mapping. $K^{*}$ denotes the adjoint of $K \in \mathcal{L}_{\mathcal{B}, \mathcal{B}^{\prime}}$ and $h \otimes h^{\prime}:=\langle h, \cdot\rangle_{\mathcal{H}} h^{\prime}$ for $h \in \mathcal{H}, h^{\prime} \in \mathcal{H}^{\prime}$. In all respects, we assume our random elements to be defined on some common probability space $(\Omega, \mathfrak{A}, \mathbb{P})$. For 
$\mathcal{B}$-valued processes $\left(X_{k}\right)_{k \in \mathbb{Z}}$ and $\left(Y_{k}\right)_{k \in \mathbb{Z}}, X_{n}=\mathrm{O}_{\mathbb{P}}\left(Y_{n}\right)($ for $n \rightarrow \infty)$ denotes that $\left(X_{k} / Y_{k}\right)_{k}$ is asymptotically P-stochastic bounded. For $p \in[1, \infty)$ we denote by $L_{\mathcal{B}}^{p}=L_{\mathcal{B}}^{p}(\Omega, \mathfrak{A}, \mathbb{P})$ the space of (classes of) $\mathcal{B}$-valued random variables $X$ with $\nu_{p, \mathcal{B}}(X):=\left(\mathbb{E}|| X \|_{\mathcal{B}}^{p}\right)^{1 / p}<\infty$, we call a process $\left(X_{k}\right)_{k \in \mathbb{Z}}$ of $\mathcal{B}$-valued random variables $L_{\mathcal{B}}^{p}$-process if $X_{k} \in L_{\mathcal{B}}^{p}$ for all $k$ and centered if $\mathbb{E}\left(X_{k}\right)=0_{\mathcal{B}}$ for all $k$ with expectation in Bochnerintegral sense, see Hsing \& Eubank (2015), p. 40-45.

The rest of this article is organized as follows. Section 2 studies the probabilistic features of our ARCH and GARCH processes. In Section 3, we introduce our parameter estimators and derive asymptotic upper bounds of the estimation errors. Section 4 summarizes the main results of this article, delineates these from similar results in other articles and gives an outline for future research. Section 5 contains all proofs.

\section{Functional ARCH and GARCH models}

We start with the definition of $F$-valued ARCH and GARCH processes where in this section $F$ stands for a separable Banach space of functions $f:[0,1] \rightarrow \mathbb{R}$ being complete w.r.t. the sup-norm $\|\cdot\|_{\infty}$ and closed w.r.t. the pointwise product $\odot$, resp. for $L^{p}[0,1]$ with $p \in[1, \infty)$. Hence, $\|\cdot\|_{F}$ is either $\|\cdot\|_{\infty}$ or the norm of $L^{p}[0,1]$ defined by $\|f\|_{L^{p}[0,1]}^{p}:=\int_{0}^{1}|f(t)|^{p} \mathrm{~d} t$ for $f \in L^{p}[0,1]$ with $\langle f, g\rangle_{L^{2}[0,1]}:=\int_{0}^{1} f(t) g(t) \mathrm{d} t$ for any $f, g \in L^{2}[0,1]$ where integration is meant w.r.t. the Lebesgue-Borel measure $\lambda$ on $[0,1]$.

Definition 2.1. Let $\mathfrak{p} \in \mathbb{N}, \mathfrak{q} \in \mathbb{N}_{0}$, let $\left(\varepsilon_{k}\right)_{k \in \mathbb{Z}}$ be an i.i.d. F-valued time series, $\delta \in F_{>0}$ and $\alpha_{i}, \beta_{j} \in \mathcal{L}_{F}$ be operators with $\alpha_{i}, \beta_{j}: F_{\geq 0} \rightarrow F_{\geq 0}$ for all $i, j$. Then, if

$$
\mathscr{X}_{k}=\varepsilon_{k} \odot \sigma_{k}, \quad \sigma_{k}^{2}=\delta+\sum_{i=1}^{\mathfrak{p}} \alpha_{i}\left(\mathscr{X}_{k-i}^{2}\right)+\sum_{j=1}^{\mathfrak{q}} \beta_{j}\left(\sigma_{k-j}^{2}\right)
$$

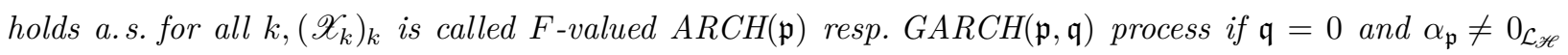
resp. if $\mathfrak{q} \in \mathbb{N}$ and $\alpha_{\mathfrak{p}} \neq 0_{\mathcal{L}_{\mathscr{H}}} \neq \beta_{\mathfrak{q}}$.

Throughout, $\alpha_{i}, \beta_{j}, \delta, \varepsilon_{k}, \mathfrak{p}, \mathfrak{q}, \sigma_{k}, \mathscr{X}_{k}$ are the variables in $(2.1)$ with $\alpha_{i}=0_{\mathcal{L}_{\mathscr{K}}}=\beta_{j}$ for $i>\mathfrak{p}, j>\mathfrak{q}$ and moreover $\mathfrak{r}:=\max (\mathfrak{p}, \mathfrak{q}), \mathfrak{s}:=\mathfrak{p}+\mathfrak{q}$. The equations in (2.1) lead to the state-space form

$$
\begin{aligned}
& \boldsymbol{\varsigma}_{k}^{(\mathfrak{p}, \mathfrak{q})}=\boldsymbol{\delta}_{k}^{(\mathfrak{p}, \mathfrak{q})}+\mathbf{\Psi}_{k}^{(\mathfrak{p}, \mathfrak{q})}\left(\boldsymbol{\varsigma}_{k-1}^{(\mathfrak{p}, \mathfrak{q})}\right) \\
& \Longleftrightarrow\left[\begin{array}{c}
\mathscr{X}_{k}^{2} \\
\mathscr{X}_{k-1}^{2} \\
\vdots \\
\mathscr{X}_{k-\mathfrak{p}+2}^{2} \\
\mathscr{X}_{k-\mathfrak{p}+1}^{2} \\
\sigma_{k}^{2} \\
\sigma_{k-1}^{2} \\
\vdots \\
\sigma_{k-\mathfrak{q}+2}^{2} \\
\sigma_{k-\mathfrak{q}+1}^{2}
\end{array}\right]=\left[\begin{array}{c}
\delta \odot \varepsilon_{k}^{2} \\
0_{F} \\
\vdots \\
0_{F} \\
0_{F} \\
\delta \\
0_{F} \\
\vdots \\
0_{F} \\
0_{F}
\end{array}\right]+\left[\begin{array}{cccccccccc}
\square_{k} \alpha_{1} & \cdots & \cdots & \cdots & \vartheta_{k} \alpha_{\mathfrak{p}} & \oslash_{k} \beta_{1} & \cdots & \cdots & \cdots & \odot_{k} \beta_{\mathfrak{q}} \\
\mathbb{I}_{F} & 0_{\mathcal{L}_{F}} & \cdots & \cdots & 0_{\mathcal{L}_{F}} & 0_{\mathcal{L}_{F}} & 0_{\mathcal{L}_{F}} & \cdots & \cdots & 0_{\mathcal{L}_{F}} \\
0_{\mathcal{L}_{F}} & \mathbb{I}_{F} & 0_{\mathcal{L}_{F}} & \cdots & 0_{\mathcal{L}_{F}} & 0_{\mathcal{L}_{F}} & \ddots & \ddots & \cdots & 0_{\mathcal{L}_{F}} \\
\vdots & \ddots & \ddots & \ddots & \vdots & \vdots & \ddots & \ddots & \ddots & \vdots \\
0_{\mathcal{L}_{F}} & \cdots & 0_{\mathcal{L}_{F}} & \mathbb{I}_{F} & 0_{\mathcal{L}_{F}} & 0_{\mathcal{L}_{F}} & \cdots & 0_{\mathcal{L}_{F}} & 0_{\mathcal{L}_{F}} & 0_{\mathcal{L}_{F}} \\
\alpha_{1} & \cdots & \cdots & \cdots & \alpha_{\mathfrak{p}} & \beta_{1} & \cdots & \cdots & \cdots & \beta_{\mathfrak{q}} \\
0_{\mathcal{L}_{F}} & 0_{\mathcal{L}_{F}} & \cdots & \cdots & 0_{\mathcal{L}_{F}} & \mathbb{I}_{F} & 0_{\mathcal{L}_{F}} & \cdots & \cdots & 0_{\mathcal{L}_{F}} \\
0_{\mathcal{L}_{F}} & \ddots & \ddots & \cdots & 0_{\mathcal{L}_{F}} & 0_{\mathcal{L}_{F}} & \mathbb{I}_{F} & 0_{\mathcal{L}_{F}} & \cdots & 0_{\mathcal{L}_{F}} \\
\vdots & \ddots & \ddots & \ddots & \vdots & \vdots & \ddots & \ddots & \ddots & \vdots \\
0_{\mathcal{L}_{F}} & \cdots & 0_{\mathcal{L}_{F}} & 0_{\mathcal{L}_{F}} & 0_{\mathcal{L}_{F}} & 0_{\mathcal{L}_{F}} & \cdots & 0_{\mathcal{L}_{F}} & \mathbb{I}_{F} & 0_{\mathcal{L}_{F}}
\end{array}\right]\left[\begin{array}{c}
\mathscr{X}_{k-1}^{2} \\
\mathscr{X}_{k-2}^{2} \\
\vdots \\
\mathscr{X}_{k-\mathfrak{p}+1}^{2} \\
\mathscr{X}_{k-\mathfrak{p}}^{2} \\
\sigma_{k-1}^{2} \\
\sigma_{k-2}^{2} \\
\vdots \\
\sigma_{k-\mathfrak{q}+1}^{2} \\
\sigma_{k-\mathfrak{q}}^{2}
\end{array}\right]
\end{aligned}
$$

with $\square_{k}: \dot{F} \rightarrow F, f \mapsto f \odot \varepsilon_{k}^{2}$ where

$$
\dot{F}:= \begin{cases}L^{2 p}, & \text { if } F=L^{p}, \\ F, & \text { if } F \text { is a Banach space w.r.t. }\|\cdot\|_{\infty} .\end{cases}
$$

Furthermore, if

$$
\mathbb{E} \ln ^{+}\left\|\varepsilon_{0}^{2}\right\|_{\dot{F}}<\infty
$$


where $\ln ^{+}(\cdot):=\ln (\max (1, \cdot))$, then $\left\|\varepsilon_{0}^{2}\right\|_{\dot{F}}<\infty$ a.s. and hence $\bullet_{k}$ is a bounded operator a.s. with

$$
\left\|\nabla_{k}\right\|_{\mathcal{L}_{\dot{F}, F}} \leq\left\|\varepsilon_{k}^{2}\right\|_{\dot{F}} \quad \text { a.s. }
$$

Consequently, $\boldsymbol{\varsigma}_{k}^{(\mathfrak{p}, \mathfrak{q})}, \boldsymbol{\delta}_{k}^{(\mathfrak{p}, \mathfrak{q})} \in F^{\mathfrak{s}}$ a.s. and we have $\Psi_{k}^{(\mathfrak{p}, \mathfrak{q})} \in \mathcal{L}_{F^{\mathfrak{s}}}$ a.s. if $\delta, \varepsilon_{k}^{2}, \sigma_{k-\mathfrak{q}}^{2}, \ldots, \sigma_{k}^{2} \in \dot{F}$ a.s. Moreover, (2.3) and $\|\cdot\|_{\mathcal{L}_{F}} \leq\|\cdot\|_{\mathcal{L}_{F, F}}$ imply (see Kühnert (2019), p.28)

$$
\mathbb{E} \ln ^{+}\left\|\Psi_{0}^{(\mathfrak{p}, \mathfrak{q})}\right\|_{\mathcal{L}_{F^{\mathfrak{s}}}}<\infty
$$

Since $\left(\mathbf{\Psi}_{k}^{(\mathfrak{p}, \mathfrak{q}}\right)_{k}$ is i.i.d. and $\|\cdot\|_{\mathcal{L}_{F^{\mathfrak{s}}}}$ is sub-multiplicative, according to Kingman (1974), Theorem 6 we have

$$
\begin{aligned}
\gamma^{(\mathfrak{p}, \mathfrak{q})} & :=\lim _{k \rightarrow \infty} \frac{1}{k} \mathbb{E} \ln \left\|\mathbf{\Psi}_{k}^{(\mathfrak{p}, \mathfrak{q})} \mathbf{\Psi}_{k-1}^{(\mathfrak{p}, \mathfrak{q})} \cdots \mathbf{\Psi}_{1}^{(\mathfrak{p}, \mathfrak{q})}\right\|_{\mathcal{L}_{F^{\mathfrak{s}}}} \\
& =\lim _{k \rightarrow \infty} \frac{1}{k} \ln \left\|\boldsymbol{\Psi}_{k}^{(\mathfrak{p}, \mathfrak{q})} \mathbf{\Psi}_{k-1}^{(\mathfrak{p}, \mathfrak{q})} \cdots \mathbf{\Psi}_{1}^{(\mathfrak{p}, \mathfrak{q})}\right\|_{\mathcal{L}_{F^{\mathfrak{s}}}} \text { a.s. }
\end{aligned}
$$

where $\gamma^{(\mathfrak{p}, \mathfrak{q})}$ is called top Lyapunov exponent of $\left(\mathbf{\Psi}_{k}^{(\mathfrak{p}, \mathfrak{q}}\right)_{k}$ with $\gamma^{(\mathfrak{p}, \mathfrak{q})} \in[-\infty, \infty)$.

Now, we can state sufficient conditions for the existence of nonanticipative, strictly stationary solutions in the $\mathrm{ARCH}$ and the GARCH model where a $F$-valued time series $\left(Y_{k}\right)_{k \in \mathbb{Z}}$ is called nonanticipative w.r.t. another $F$-valued time series $\left(\varepsilon_{k}\right)_{k \in \mathbb{Z}}$ if there is a measurable function $f: F^{\infty} \rightarrow F$ such that

$$
Y_{k}=f\left(\varepsilon_{k}, \varepsilon_{k-1}, \ldots\right)
$$

holds a.s. for all $k$. If $\left(\varepsilon_{k}\right)_{k \in \mathbb{Z}}$ is strictly stationary and ergodic, which is especially the case if $\left(\varepsilon_{k}\right)_{k}$ is i.i.d., then (2.8) implies that $\left(Y_{k}\right)_{k}$ is also strictly stationary and ergodic after Stout (1974), Theorem 3.5.8.

Theorem 2.1. Let the assumptions in Definition 2.1, $\delta \in \dot{F}_{>0}$ and $\alpha_{i}, \beta_{j} \in \mathcal{L}_{F, \dot{F}}$ for all $i, j$ hold.

(a) If

$$
\gamma^{(\mathfrak{p}, \mathfrak{q})}<0
$$

then the equations in (2.1) have a unique, strictly stationary, nonanticipative w.r.t. $\left(\varepsilon_{k}\right)_{k}$ and ergodic solution where $\sigma_{k}^{2}=f\left(\varepsilon_{k-1}, \varepsilon_{k-2}, \ldots\right)$ a.s. for all $k$ for some measurable function $f: F^{\infty} \rightarrow F$.

(b) If there are $n \in \mathbb{N}$ and $\nu>0$ such that

$$
\psi_{n, \nu}^{(\mathfrak{p}, \mathfrak{q})}:=\mathbb{E}\left\|\Psi_{n}^{(\mathfrak{p}, \mathfrak{q})} \mathbf{\Psi}_{n-1}^{(\mathfrak{p}, \mathfrak{q})} \cdots \mathbf{\Psi}_{1}^{(\mathfrak{p}, \mathfrak{q})}\right\|_{\mathcal{L}_{F^{\mathfrak{s}}}}^{\nu}<1
$$

then (2.9) holds.

Though (2.10) is stricter than (2.9), it is easier to show. Furthermore, (2.10) is useful for the simulation of an initial value of $F$-valued $\mathrm{ARCH}$ and $\mathrm{GARCH}$ processes, as we can see in the following.

Corollary 2.1. Let (2.10) hold for some $n \in \mathbb{N}$ and $\nu>0$. Further, define $\tilde{\boldsymbol{\varsigma}}_{k}^{(\mathfrak{p}, \mathfrak{q})}:=\boldsymbol{\delta}_{k}^{(\mathfrak{p}, \mathfrak{q})}+\mathbf{\Psi}_{k}^{(\mathfrak{p}, \mathfrak{q})}\left(\tilde{\boldsymbol{\varsigma}}_{k-1}^{(\mathfrak{p}, \mathfrak{q})}\right)$ for $k \in \mathbb{N}$, where $\tilde{\boldsymbol{\varsigma}}_{0}^{(\mathfrak{p}, \mathfrak{q})} \in F^{\mathfrak{s}}$ is some deterministic value. Then, there is some $\rho \in(0,1)$ with

$$
\mathbb{E}\left\|\boldsymbol{\varsigma}_{N}^{(\mathfrak{p}, \mathfrak{q})}-\tilde{\boldsymbol{\varsigma}}_{N}^{(\mathfrak{p}, \mathfrak{q})}\right\|_{F^{\mathfrak{s}}}^{\nu}=\mathrm{O}\left(\rho^{N}\right)
$$

Based on ideas in Aue et al. (2017), Hörmann et al. (2013) and with (2.10), we derive a sufficient condition for the existence of moments and for weak dependence, to be precise $L^{p}$ - $m$-approximibility, of $F$-valued $\operatorname{ARCH}(\mathfrak{p})$ and $\operatorname{GARCH}(\mathfrak{p}, \mathfrak{q})$ processes for any $\mathfrak{p}, \mathfrak{q} \in \mathbb{N}$. Finite moments and $L^{p}$-m-approximibility are used to estimate the $\mathrm{ARCH}$ and $\mathrm{GARCH}$ parameters. An $F$-valued time series $\left(Y_{k}\right)_{k \in \mathbb{Z}}$ is called $L_{F}^{p}-m$ approximable for $p \geq 1$ if $Y_{k}=f\left(\varepsilon_{k}, \varepsilon_{k-1}, \ldots\right)$ a.s. for all $k$ for an i.i.d. time series $\left(\varepsilon_{k}\right)_{k \in \mathbb{Z}}$ and a measurable function $f: F^{\infty} \rightarrow F$ (thus $\left(Y_{k}\right)_{k}$ is nonanticipative w.r.t. $\left.\left(\varepsilon_{k}\right)_{k}\right)$ and if

$$
\sum_{m=1}^{\infty} \nu_{p, \mathcal{B}}\left(Y_{m}-Y_{m}^{(m)}\right)<\infty
$$


holds where $\nu_{p, \mathcal{B}}(\cdot)=\left(\mathbb{E}\|\cdot\|_{\mathcal{B}}^{p}\right)^{1 / p}$ and $Y_{k}^{(m)}:=f\left(\varepsilon_{k}, \varepsilon_{k-1}, \ldots, \varepsilon_{k-m+1}, \varepsilon_{k-m}^{(k)}, \varepsilon_{k-m-1}^{(k)}, \ldots\right)$ for all $k, m$ with independent copies $\left(\varepsilon_{k}^{(n)}\right)_{k \in \mathbb{Z}}$ of $\left(\varepsilon_{k}\right)_{k \in \mathbb{Z}}$ for all $n$. For each $m$, the sequences $\left(Y_{k}^{(m)}\right)_{k \in \mathbb{Z}}$ are strictly stationary, $m$-dependent and each $Y_{k}^{(m)}$ equals $Y_{k}$ in distribution. Moreover, $\left(Y_{k}\right)_{k \in \mathbb{Z}}$ is called geometrically $L_{F}^{p}-m$ approximable if $\left(Y_{k}\right)_{k}$ is $L_{F}^{p}-m$-approximable and if there exists a $\rho \in(0,1)$ with $\nu_{p, \mathcal{B}}\left(Y_{m}-Y_{m}^{(m)}\right)=\mathrm{O}\left(\rho^{m}\right)$. For a detailed introduction to $L^{p}-m$-approximibility, see Hörmann \& Kokoszka (2010).

Lemma 2.1. Let $\mathbb{E}\left\|\varepsilon_{0}^{2}\right\|_{\dot{F}}^{\nu}<\infty$. Also, let (2.10) hold for some $\nu>0$ and $n \in \mathbb{N}$. Then

(a) $\mathbb{E}\left\|\mathscr{X}_{0}^{2}\right\|_{F}^{\nu}<\infty$ and $\mathbb{E}\left\|\sigma_{0}^{2}\right\|_{\dot{F}}^{\nu}<\infty$

(b) $\left(\mathscr{X}_{k}^{2}\right)_{k}$ is geometrically $L_{F}^{\nu}$-m-approximable and $\left(\sigma_{k}^{2}\right)_{k}$ is geometrically $L_{\dot{F}}^{\nu}$-m-approximable.

\section{Estimation}

In this section, we establish estimators for the parameters of $\mathscr{H}$-valued ARCH and GARCH processes with known orders where $\mathscr{H}:=L^{2}[0,1]$ and deduce consistency results for their estimation errors. Throughout the section, we write $\dot{\mathscr{H}}:=L^{4}[0,1]$ and, except for in section 3.1 , we impose the following.

Assumption 3.1. $\delta \in \dot{\mathscr{H}}_{>0}, \alpha_{i}, \beta_{j} \in \mathcal{S}_{\mathscr{H}} \cap \mathcal{L}_{\mathscr{H}, \mathscr{H}}$ for all $i$ and $j, \mathbb{E}\left\|\varepsilon_{0}^{2}\right\|_{\mathscr{\mathscr { C }}}^{4}<\infty$,

$$
\mathbb{E}\left(\varepsilon_{0}^{2}(t)\right)=1
$$

for $\lambda$-a.e. $t \in[0,1]$ and there are $n \in \mathbb{N}$ and $\nu=4$ with (2.10).

Assumption 3.1 implies $\mathbb{E}\left(\mathscr{X}_{k}^{2}(t)\right)=\mathbb{E}\left(\sigma_{k}^{2}(t)\right)$ for $\lambda$-a.e. $t$ and all $k$. Thus, $(2.1)$ yields

$$
\mathscr{Z}_{k}=\nu_{k}+\sum_{i=1}^{\mathfrak{r}}\left(\alpha_{i}+\beta_{i}\right)\left(\mathscr{Z}_{k-i}\right)+\sum_{j=1}^{\mathfrak{q}}\left(-\beta_{j}\right)\left(\nu_{k-j}\right)
$$

a.s. for all $k$ where $\alpha_{i}=0_{\mathcal{L}_{\mathscr{H}}}=\beta_{j}$ for $i>\mathfrak{p}, j>\mathfrak{q}, \mathscr{Z}_{k}:=\mathscr{X}_{k}^{2}-m_{2}$ with $m_{2}:=\mathbb{E}\left(\mathscr{X}_{1}^{2}\right)$ and $\nu_{k-j}:=$ $\mathscr{X}_{k-j}^{2}-\sigma_{k-j}^{2}$. Hence, $\mathscr{Z}=\left(\mathscr{Z}_{k}\right)_{k}$ is a $\mathscr{H}$-valued $\operatorname{AR}(\mathfrak{p})$ resp. $\operatorname{ARMA}(\mathfrak{r}, \mathfrak{q})$ process if $\mathfrak{q}=0$ resp. $\mathfrak{q} \in \mathbb{N}$ with time series of innovations $\boldsymbol{\nu}:=\left(\nu_{k}\right)_{k}$ which is not i.i.d. but stationary. Moreover, both $\mathscr{Z}$ and $\boldsymbol{\nu}$ are centered, stationary, nonanticipative w.r.t. $\left(\varepsilon_{k}\right)_{k}$ and geometrically $L_{\mathscr{H}}^{4}$-m-approximable.

For the estimation of the operators in (2.1), we use (3.2) and we impose the following.

Assumption 3.2. $\delta \in \ell^{\infty}[0,1], \alpha_{i}, \beta_{j} \in \mathcal{L}_{\mathscr{H}, \ell^{\infty}[0,1]}$ for all $i$ and $j,\left\|\Gamma_{\mathfrak{p}, \mathfrak{q}}\right\|_{\mathcal{L}_{\mathscr{H}}}<1$ where $\Gamma_{\mathfrak{p}, \mathfrak{q}}:=\sum_{i=1}^{\mathfrak{p}} \alpha_{i}+$ $\sum_{j=1}^{\mathfrak{q}} \beta_{j}$ and there is no closed, affine subspace $U \subsetneq \mathscr{H}$ with $\mathbb{P}\left(\varepsilon_{0}^{2} \in U\right)=1$.

Lemma 3.1. Let Assumptions 3.1-3.2 hold. Then, there is no closed subspace $V \subsetneq \mathscr{H}$ with $\mathbb{P}\left(\mathscr{X}_{0}^{2} \in V\right)=$ $1, \mathbb{P}\left(\nu_{0} \in V\right)=1, \mathbb{P}\left(\mathscr{Z}_{0} \in V\right)=1$ and the operators $\mathscr{C}_{0 ; \varepsilon^{2}}, \mathscr{C}_{0 ; \mathscr{X}}, \mathscr{C}_{0 ; \nu}$ and $\mathscr{C}_{0 ; \mathscr{Z}}$ are injective.

\subsection{Preliminaries}

Here, in order to estimate the parameters in (2.1), we state certain assumptions and establish various convergence results dealing with the asymptotic behaviour of estimation errors of specific eigenvalues and expected values, operators and eigenfunctions in Hilbert spaces $\left(\mathcal{H},\langle\cdot, \cdot\rangle_{\mathcal{H}}\right),\left(\mathcal{H}^{\prime},\langle\cdot, \cdot\rangle_{\mathcal{H}^{\prime}}\right)$ and $\left(\mathcal{H}^{\prime \prime},\langle\cdot, \cdot\rangle_{\mathcal{H}^{\prime \prime}}\right)$. We also deal with the estimation of operators within a composition of operators.

\subsubsection{Estimation of expected values, lag- $h$-covariance operators and other operators}

Firstly, we define lag- $h$-covariance operators and their empirical versions. 
Definition 3.1. Let $\boldsymbol{X}=\left(X_{k}\right)_{k \in \mathbb{Z}}$ be a stationary $L_{\mathcal{H}}^{2}$-valued time series and let $h \in \mathbb{Z}$. Then, the lag-hcovariance operator of $\boldsymbol{X}$ is defined by

$$
\mathscr{C}_{h}=\mathscr{C}_{h ; \boldsymbol{X}}:=\mathbb{E}\left[\left(X_{0}-m_{1}\right) \otimes\left(X_{h}-m_{1}\right)\right]
$$

where $m_{1}=m_{1}(\boldsymbol{X}):=\mathbb{E}\left(X_{1}\right)$ and the empirical lag-h-covariance operator of $\boldsymbol{X}$ is defined by

$$
\hat{\mathscr{C}}_{h}=\hat{\mathscr{C}}_{h ; \boldsymbol{X}}:=\left\{\begin{array}{lr}
\frac{1}{N_{h}-1} \sum_{k=|h|+1}^{N_{h}}\left(X_{k}-\hat{m}_{1}\right) \otimes\left(X_{k+h}-\hat{m}_{1}\right), & 1-N<h<0, \\
\frac{1}{N_{h}-1} \sum_{k=1}^{N_{h}}\left(X_{k}-\hat{m}_{1}\right) \otimes\left(X_{k+h}-\hat{m}_{1}\right), & 0 \leq h<N-1
\end{array}\right.
$$

where $\hat{m}_{1}=\hat{m}_{1}(\boldsymbol{X}):=N_{h}^{-1} \sum_{i=1}^{N_{h}} X_{i}$ and $N_{h}:=N-|h|$ with $N \in \mathbb{N}$ and $|h|<N-1$. The operators $\mathscr{C}_{0}$ and $\hat{\mathscr{C}}_{0}$ are also called covariance operator resp. empirical covariance operator.

$\hat{\mathscr{C}}_{h}$ are bounded operators with finite-dimensional image with $\hat{\mathscr{C}}_{h}^{*}=\hat{\mathscr{C}}_{-h}$ for all $h$. Furthermore, $\hat{\mathscr{C}}_{0}$ is selfadjoint and positive semi-definite. We obtain the following convergence rates.

Lemma 3.2. Let $\boldsymbol{X}=\left(X_{k}\right)_{k \in \mathbb{Z}}$ be a $L_{\mathcal{H}}^{4}$-m-approximable time series. Then

$$
\hat{m}_{l}=\hat{m}_{l}(\boldsymbol{X}):=N^{-1} \sum_{i=1}^{N} X_{i}^{l}
$$

is an unbiased esimator for $m_{l}=m_{l}(\boldsymbol{X}):=\mathbb{E}\left(X_{1}^{l}\right)$ for any $l=1,2$ and $N \in \mathbb{N}$ with

$$
\mathbb{E}\left\|\hat{m}_{l}-m_{l}\right\|_{\mathcal{H}}^{2}=\mathrm{O}\left(N^{-1}\right) .
$$

Theorem 3.1. Let $\boldsymbol{X}=\left(X_{k}\right)_{k \in \mathbb{Z}}$ be a $L_{\mathcal{H}}^{4}$-m-approximable time series. Then

$$
\left\|\hat{\mathscr{C}}_{h}-\mathscr{C}_{h}\right\|_{\mathcal{S}_{\mathcal{H}}}^{2}= \begin{cases}\mathrm{O}_{\mathbb{P}}\left(N^{-1}\right), & \text { if } h \in \mathbb{Z} \text { is fixed } \\ \mathrm{O}_{\mathbb{P}}\left(h N^{-1}\right), & \text { if } h=h_{N}=\Xi(1, N) .\end{cases}
$$

Remark 3.1. Kühnert (2019), Theorem 4.4 states the assertion of Theorem 3.1 also for convergence in mean for centered time series, and thus generalizes Hörmann $\&$ Kokoszka (2010), Theorem 3.1

Based on ideas in Aue \& Klepsch (2017), for centered time series $\boldsymbol{X}=\left(X_{k}\right)_{k \in \mathbb{Z}}$ we define the operators

$$
\mathfrak{S}_{d, m}=\mathscr{C}_{X_{d}(d), X_{d+m}}:=\mathbb{E}\left[X_{d}(d) \otimes X_{d+m}\right] \quad \text { and } \quad \mathfrak{S}_{d}:=\mathscr{C}_{0 ; \boldsymbol{X}(d)}=\mathbb{E}\left[X_{d}(d) \otimes X_{d}(d)\right]
$$

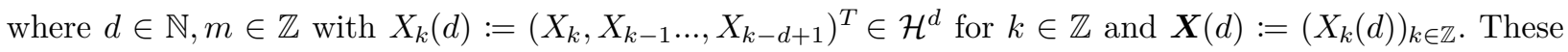
operators satisfy $\mathfrak{S}_{d, m} \in \mathcal{N}_{\mathcal{H}^{d}, \mathcal{H}}$ and $\mathfrak{S}_{d} \in \mathcal{N}_{\mathcal{H}^{d}}$ as well as (see Kühnert (2019), p.56)

$$
\left\|\mathfrak{S}_{d, m}\right\|_{\mathcal{N}_{\mathcal{H}^{d}, \mathcal{H}}} \leq \sqrt{d} \mathbb{E}\left\|X_{0}\right\|_{\mathcal{H}}^{2} \quad \text { and } \quad\left\|\mathfrak{S}_{d}\right\|_{\mathcal{N}_{\mathcal{H}^{d}}}=d \mathbb{E}\left\|X_{0}\right\|_{\mathscr{H}}^{2}
$$

Also, given a sample $X_{1}, \ldots, X_{N}$ of $\boldsymbol{X}$ with $N>d$, the operators

$$
\hat{\mathfrak{S}}_{d, 1}:=\frac{1}{N_{d}-1} \sum_{k=1}^{N_{d}}\left(X_{k+d-1}^{2}(d)-\hat{m}_{1}\left(\boldsymbol{X}^{2}(d)\right)\right) \otimes\left(X_{k+d}^{2}-\hat{m}_{1}\left(\boldsymbol{X}^{2}\right)\right),
$$

where $N_{d}:=N-d, \hat{m}_{1}\left(\boldsymbol{X}^{2}(d)\right):=N_{d}^{-1} \sum_{i=1}^{N_{d}} X_{d+i-1}^{2}(d)$ and $\hat{m}_{1}\left(\boldsymbol{X}^{2}\right):=N_{d}^{-1} \sum_{j=1}^{N_{d}} X_{d+j}^{2}$, satisfy (see Kühnert (2019), Definition and properties 4.36)

$$
\left\|\hat{\mathfrak{S}}_{d, 1}-\mathfrak{S}_{d, 1}\right\|_{\mathcal{S}_{\mathcal{H}^{d}, \mathcal{H}}}^{2}= \begin{cases}\mathrm{O}_{\mathbb{P}}\left(N^{-1}\right), & \text { if } d \in \mathbb{N} \text { is fixed, } \\ \mathrm{O}_{\mathbb{P}}\left(d^{2} N^{-1}\right), & \text { if } d=d_{N}=\Xi(1, N) .\end{cases}
$$

Moreover, the empirical covariance operators

$$
\hat{\mathfrak{S}}_{d}:=\frac{1}{N_{d}-1} \sum_{k=1}^{N_{d}}\left(X_{d+k-1}^{2}(d)-\hat{m}_{1}\left(\boldsymbol{X}^{2}(d)\right)\right) \otimes\left(X_{d+k-1}^{2}(d)-\hat{m}_{1}\left(\boldsymbol{X}^{2}(d)\right)\right)
$$

satisfy

$$
\left\|\hat{\mathfrak{S}}_{d}-\mathfrak{S}_{d}\right\|_{\mathcal{S}_{\mathcal{H}^{d}}}^{2}= \begin{cases}\mathrm{O}_{\mathbb{P}}\left(N^{-1}\right), & \text { if } d \in \mathbb{N} \text { is fixed, } \\ \mathrm{O}_{\mathbb{P}}\left(d^{3} N^{-1}\right), & \text { if } d=d_{N}=\Xi(1, N) .\end{cases}
$$




\subsubsection{Estimation of eigenvalues and eigenfunctions}

Here, we derive asymptotic upper bounds of the estimation errors of the eigenvalues and eigenfunctions of a compact, self-adjoint and positive semi-definite operator $\mathscr{K} \in \mathcal{K}_{\mathcal{H}}$, estimated by a sequence $\left(\hat{\mathscr{K}}_{N}\right)_{N \in \mathbb{N}} \subseteq \mathcal{K}_{\mathcal{H}}$ of compact, self-adjoint, positive semi-definite operators, where each $\hat{\mathscr{K}}_{N}$ depends on $N$ observations of a stationary time series $\boldsymbol{X}=\left(X_{k}\right)_{k \in \mathbb{Z}}$. Further, $\left(\mathfrak{k}_{j}\right)_{j \in \mathbb{N}}$ resp. $\left(\hat{\mathfrak{k}}_{j}\right)_{j \in \mathbb{N}}$ are the eigenfunction sequences and $\left(k_{j}\right)_{j \in \mathbb{N}}$ resp. $\left(\hat{k}_{j}\right)_{j \in \mathbb{N}}$ the associated w.l.o.g. monotonically decreasing eigenvalue sequences of $\mathscr{K}$ resp. $\hat{\mathscr{K}}_{N}$. For asymptotic upper bounds of the estimation errors of the eigenvalues and eigenfunctions, we need

$$
\left|a_{j}-b_{j}\right| \leq\|A-B\|_{\mathcal{L}_{\mathcal{H}}}, \quad j \in \mathbb{N} .
$$

This is true according to Bosq (2000), Lemma 4.2 where $A, B \in \mathcal{K}_{\mathcal{H}}$ are compact operators with singular value decompositions $A=\sum_{j=1}^{\infty} a_{j}\left(\mathfrak{a}_{j} \otimes \mathfrak{a}_{j}^{\prime}\right)$ resp. $B=\sum_{j=1}^{\infty} b_{j}\left(\mathfrak{b}_{j} \otimes \mathfrak{b}_{j}^{\prime}\right)$.

Corollary 3.1. Let $\left\|\hat{\mathscr{K}}_{N}-\mathscr{K}\right\|_{\mathcal{L}_{\mathcal{H}}}^{2}=\mathrm{O}_{\mathbb{P}}\left(a_{N}\right)$ hold where $a_{N}=\Xi\left[N^{-1}, 1\right)$. Then

$$
\sup _{j \in \mathbb{N}}\left(\hat{k}_{j}-k_{j}\right)^{2}=\mathrm{O}_{\mathbb{P}}\left(a_{N}\right) .
$$

Moreover, if $k_{b_{N}}=\Xi\left[\sqrt{a_{N}}, 1\right]$ holds where $b_{N}=\Omega(1)$, then

$$
\hat{k}_{b_{N}}=\mathrm{O}_{\mathbb{P}}\left(k_{b_{N}}\right) \quad \text { and } \quad k_{b_{N}}=\mathrm{O}_{\mathbb{P}}\left(\hat{k}_{b_{N}}\right) .
$$

Because the eigenfunctions of $\hat{\mathscr{K}}_{N}$ are unambiguously determined except for their sign,

$$
\hat{\mathfrak{k}}_{j}^{\prime}:=\operatorname{sgn}\left(\left\langle\hat{\mathfrak{k}}_{j}, \mathfrak{k}_{j}\right\rangle_{\mathcal{H}}\right) \hat{\mathfrak{k}}_{j}
$$

can be used as an estimator for $\mathfrak{k}_{j}$ if $\hat{\mathfrak{k}}_{j} \not \perp \mathfrak{k}_{j}$ a.s. holds where sgn is the signum function. According to Bosq (2000), Lemma 4.3, which can be generalized to any compact, self-adjoint and positive semi-definite operators,

$$
\left\|\hat{\mathfrak{k}}_{j}^{\prime}-\mathfrak{k}_{j}\right\|_{\mathcal{H}} \leq \tilde{\gamma}_{j}\left\|\hat{\mathscr{K}}_{N}-\mathscr{K}\right\|_{\mathcal{L}_{\mathcal{H}}}, \quad j \in \mathbb{N},
$$

if the eigenspace of $k_{j}$ is one-dimensional, where $\tilde{\gamma}_{1}:=2 \sqrt{2} \gamma_{1}, \tilde{\gamma}_{j}:=2 \sqrt{2} \max \left(\gamma_{j-1}, \gamma_{j}\right)$ for $j>1$ and $\gamma_{j}:=\left(k_{j}-k_{j+1}\right)^{-1}$ for $j \in \mathbb{N}$. The problem in using $\hat{\mathfrak{k}}_{j}^{\prime}$ as an estimator for $\mathfrak{k}_{j}$ is, that $\hat{\mathfrak{k}}_{j} \not \perp \mathfrak{k}_{j}$ a.s. and thus $\operatorname{sgn}\left(\left\langle\hat{\mathfrak{k}}_{j}, \mathfrak{k}_{j}\right\rangle_{\mathcal{H}}\right) \neq 0$ a.s., which is needed to obtain asymptotic upper bounds of the estimation errors of the operators in the $\mathscr{H}$-valued ARCH and GARCH model, is not guaranteed for all $j, N$. Therefore, we modify $\hat{\mathfrak{k}}_{j}$ in the following way. Let $\left(h_{j}\right)_{j \in \mathbb{N}}$ be a CONS of $\mathcal{H}$ and let $\left(\zeta_{j}\right)_{j \in \mathbb{N}}$ be a sequence of i.i.d. and $\mathcal{N}(0,1)$-distributed random variables, independent of the observations of $\boldsymbol{X}$. Then

$$
\hat{\mathfrak{k}}_{j}^{\prime \prime}:=\hat{\mathfrak{k}}_{j}+\sum_{i=1}^{\infty} \frac{\zeta_{i} h_{i}}{i^{2} N}
$$

is well-defined for all $j, N$ with $\hat{\mathfrak{k}}_{j}^{\prime \prime} \not \perp \mathfrak{k}_{j}$ a.s. and in consequence $\operatorname{sgn}\left(\left\langle\hat{\mathfrak{k}}_{j}^{\prime \prime}, \mathfrak{k}_{j}\right\rangle_{\mathcal{H}^{\prime}}\right) \neq 0$ a.s. Thus we use

$$
\hat{\mathfrak{k}}_{j}^{\prime \prime \prime}:=\operatorname{sgn}\left(\left\langle\hat{\mathfrak{k}}_{j}^{\prime \prime}, \mathfrak{k}_{j}\right\rangle_{\mathcal{H}^{\prime}}\right) \hat{\mathfrak{k}}_{j}
$$

as an estimator for $\mathfrak{k}_{j}$, where $\left(\hat{\mathfrak{k}}_{j}^{\prime \prime \prime}\right)_{j}$ is a CONS of $\mathcal{H}$ a.s. according to the spectral theorem.

Assumption 3.3. For all $j, k_{j} \neq k_{j+1}$ and $\kappa(j)=k_{j}$ holds where $\kappa: \mathbb{R} \rightarrow \mathbb{R}$ is a convex function.

If $\mathscr{K}$ is injective and if the eigenvalues of $\mathscr{K}$ satisfy Assumption 3.3 , then

$$
k_{1}>k_{2}>\cdots>0 .
$$

Moreover, for any sequence $m=m_{N}=\Omega(1)$ :

$$
\sup _{j \leq m} \tilde{\gamma}_{j}=\gamma_{m} \asymp k_{m}^{-1} .
$$


Lemma 3.3. Let $\mathscr{K}$ be injective, let Assumption 3.3 and $\| \hat{\mathscr{K}}_{N}-\mathscr{K}_{\mathcal{L}_{\mathcal{H}}}^{2}=\mathrm{O}_{\mathbb{P}}\left(a_{N}\right)$ with $a_{N}=\Xi\left[N^{-1}, 1\right)$ hold. Then

$$
\left\|\hat{\mathfrak{k}}_{j}^{\prime \prime \prime}-\mathfrak{k}_{j}\right\|_{\mathcal{H}}^{2}=\mathrm{O}_{\mathbb{P}}\left(a_{N}\right), \quad j \in \mathbb{N} .
$$

Furthermore, if $k_{m}=\omega\left(\sqrt{a_{N}}\right)$ holds where $m=m_{N}=\Xi[1, N)$, then

$$
\sup _{j \leq m}\left\|\hat{\mathfrak{k}}_{j}^{\prime \prime \prime}-\mathfrak{k}_{j}\right\|_{\mathcal{H}}^{2}=\mathrm{O}_{\mathbb{P}}\left(k_{m}^{-2} a_{N}\right) .
$$

\subsubsection{Some notes on estimating operators}

In this paper, we estimate bounded operators $B \in \mathcal{L}_{\mathcal{H}^{\prime}, \mathcal{H}^{\prime \prime}}$ in equations as

$$
A=B C
$$

where $A \in \mathcal{L}_{\mathcal{H}, \mathcal{H}^{\prime \prime}}$ and $C \in \mathcal{L}_{\mathcal{H}, \mathcal{H}^{\prime}}$. Identifiability of $B$ from (3.24), that is $B C=\tilde{B} C$ implying $B=\tilde{B}$, is only guaranteed if $B$ has dense image. Further, if $C$ is a compact operator and thus has no bounded inverse, we use the tikhonov-regularized of $C$ in order to isolate $B$. Also, when estimating operators without projecting them on a finite-dimensional subspace, we impose the following Sobolev condition.

Assumption 3.4. Let $S \in \mathcal{S}_{\mathcal{H}, \mathcal{H}^{\prime}}$ and let $\left(\phi_{i j}\right)_{i, j \in \mathbb{N}}$ be a CONS of $\mathcal{S}_{\mathcal{H}, \mathcal{H}^{\prime}}$. Then, $\left(S,\left(\phi_{i j}\right)_{i, j}\right)$ satisfies the Sobolev condition for $\beta>0$ if

$$
\sum_{i, j=1}^{\infty}\left\langle S, \phi_{i j}\right\rangle_{\mathcal{S}_{\mathcal{H}, \mathcal{H}^{\prime}}}^{2}\left(1+i^{2 \beta}+j^{2 \beta}\right)<\infty
$$

This identity implies with $\mathscr{J}_{m}^{c}:=\left\{\phi_{i j} \mid i, j \in \mathbb{N}, i \vee j>m\right\}$ for $m=m_{N} \rightarrow \infty$ :

$$
\left\|\coprod_{\mathscr{J}_{m}^{c}} S\right\|_{\mathcal{S}_{\mathcal{H}, \mathcal{H}^{\prime}}}^{2}=\sum_{\substack{i, j \in \mathbb{N} \\ i \vee j>m}}\left\langle S, \phi_{i j}\right\rangle_{\mathcal{S}_{\mathcal{H}, \mathcal{H}^{\prime}}}^{2} \leq\left(1+m^{2 \beta}\right)^{-1} \sum_{i, j=1}^{\infty}\left\langle S, \phi_{i j}\right\rangle_{\mathcal{S}_{\mathcal{H}, \mathcal{H}^{\prime}}}^{2}\left(1+i^{2 \beta}+j^{2 \beta}\right)=\mathrm{O}\left(m^{-2 \beta}\right) .
$$

\subsection{Estimation of $\delta$ in the functional ARCH and GARCH model}

We derive an estimator of $\delta$ in $\mathscr{H}$-valued $\operatorname{ARCH}(\mathfrak{p})$ and $\operatorname{GARCH}(\mathfrak{p}, \mathfrak{q})$ processes with $\mathfrak{p}, \mathfrak{q} \in \mathbb{N}$ from the idea of estimating $\delta$ in $\mathscr{H}$-valued $\mathrm{ARCH}(1)$ processes in Hörmann et al. (2013). Under Assumption 3.1, taking the expected value on both sides of the right equation in (2.1), yields

$$
\delta=m_{2}-\sum_{i=1}^{\mathfrak{r}}\left(\alpha_{i}+\beta_{i}\right)\left(m_{2}\right),
$$

where $\alpha_{i}=0_{\mathcal{L}_{\mathscr{H}}}=\beta_{j}$ for $i>\mathfrak{p}, j>\mathfrak{q}$. Therefore, we propose

$$
\hat{\delta}:=\hat{m}_{2}-\sum_{i=1}^{\mathfrak{r}}\left(\hat{\alpha}_{i}+\hat{\beta}_{i}\right)\left(\hat{m}_{2}\right)
$$

as an estimator for $\delta$ where $\hat{\alpha}_{i}, \hat{\beta}_{j}$ are estimators for $\alpha_{i}, \beta_{j}$ and where $\hat{m}_{2}:=N^{-1} \sum_{i=1}^{N} \mathscr{X}_{i}^{2}$.

Theorem 3.2. Let Assumption 3.1 hold. Then,

$$
\|\hat{\delta}-\delta\| \|_{\mathscr{H}}=\mathrm{O}_{\mathbb{P}}\left(N^{-1 / 2}\right)+\sum_{i=1}^{\mathfrak{r}} \mathrm{O}_{\mathbb{P}}\left(\left\|\hat{\alpha}_{i}-\alpha_{i}\right\|_{\mathcal{L}_{\mathscr{H}}}\right)+\mathrm{O}_{\mathbb{P}}\left(\left\|\hat{\beta}_{i}-\beta_{i}\right\|_{\mathcal{L}_{\mathscr{H}}}\right) .
$$




\subsection{Operator estimation in the functional ARCH model}

In the following, $\mathscr{X}:=\left(\mathscr{X}_{k}\right)_{k \in \mathbb{Z}}$ is a $\mathscr{H}$-valued $\mathrm{ARCH}(\mathfrak{p})$ process with $\mathfrak{p} \in \mathbb{N}$. Under Assumption 3.1, $\mathscr{Z}:=\left(\mathscr{Z}_{k}\right)_{k \in \mathbb{Z}}=\left(\mathscr{X}_{k}^{2}-m_{2}\right)_{k \in \mathbb{Z}}$ with $m_{2}:=\mathbb{E}\left(\mathscr{X}_{1}^{2}\right)$ is a $\mathscr{H}$-valued $\mathrm{AR}(\mathfrak{p})$ process with time series of innovations $\boldsymbol{\nu}:=\left(\nu_{k}\right)_{k \in \mathbb{Z}}=\left(\mathscr{X}_{k}^{2}-\sigma_{k}^{2}\right)_{k \in \mathbb{Z}}\left(\right.$ see p.5). Furthermore, $\mathscr{Z}(\mathfrak{p}):=\left(\mathscr{Z}_{k}(\mathfrak{p})\right)_{k \in \mathbb{Z}}$ satisfies

$$
\mathscr{Z}_{k}(\mathfrak{p})=\tilde{\nu}_{k}(\mathfrak{p})+\boldsymbol{A}_{1}\left(\mathscr{Z}_{k-1}(\mathfrak{p})\right): \Longleftrightarrow\left[\begin{array}{c}
\mathscr{Z}_{k} \\
\mathscr{Z}_{k-1} \\
\vdots \\
\mathscr{Z}_{k-\mathfrak{p}+2} \\
\mathscr{Z}_{k-\mathfrak{p}+1}
\end{array}\right]=\left[\begin{array}{c}
\nu_{k} \\
0_{\mathscr{H}} \\
\vdots \\
0_{\mathscr{H}} \\
0_{\mathscr{H}}
\end{array}\right]+\left[\begin{array}{ccccc}
\alpha_{1} & \cdots & \cdots & \cdots & \alpha_{\mathfrak{p}} \\
\mathbb{I}_{\mathscr{H}} & 0_{\mathcal{L}_{\mathscr{H}}} & \cdots & \cdots & 0_{\mathcal{L}_{\mathscr{H}}} \\
0_{\mathcal{L}_{\mathscr{H}}} & \mathbb{I}_{\mathscr{H}} & 0_{\mathcal{L}_{\mathscr{H}}} & \cdots & 0_{\mathcal{L}_{\mathscr{H}}} \\
\vdots & \ddots & \ddots & \ddots & \vdots \\
0_{\mathcal{L}_{\mathscr{H}}} & \cdots & 0_{\mathcal{L}_{\mathscr{H}}} & \mathbb{I}_{\mathscr{H}} & 0_{\mathcal{L}_{\mathscr{H}}}
\end{array}\right]\left[\begin{array}{c}
\mathscr{Z}_{k-1} \\
\mathscr{Z}_{k-2} \\
\vdots \\
\mathscr{Z}_{k-\mathfrak{p}+1} \\
\mathscr{Z}_{k-\mathfrak{p}}
\end{array}\right]
$$

a.s. for all $k$. The operator

$$
\boldsymbol{\alpha}_{[\mathfrak{p}]}:=\left[\alpha_{1} \cdots \alpha_{\mathfrak{p}}\right]
$$

is an element of $\mathcal{S}_{\mathscr{C}}$, $\mathscr{H}$ and satisfies the Yule-Walker equation

$$
\mathfrak{S}_{\mathfrak{p}, 1}=\boldsymbol{\alpha}_{[\mathfrak{p}]} \mathfrak{S}_{\mathfrak{p}}
$$

where $\mathfrak{S}_{\mathfrak{p}, 1}=\mathscr{C}_{\mathscr{Z}_{\mathfrak{p}}(\mathfrak{p}), \mathscr{Z}_{\mathfrak{p}+1}}$ and $\mathfrak{S}_{\mathfrak{p}}=\mathscr{C}_{0 ; \mathscr{Z}(\mathfrak{p})}$. Since $\mathfrak{S}_{\mathfrak{p}}$ is injective as a consequence of Lemma 3.1 (see Kühnert (2019), Lemma 4.35), $\boldsymbol{\alpha}_{[\mathfrak{p}]}$ can be identified from (3.31) and as an estimator we thus impose

$$
\hat{\boldsymbol{\alpha}}_{[\mathfrak{p}]}:=\hat{\mathfrak{S}}_{\mathfrak{p}, 1} \hat{\mathfrak{S}}_{\mathfrak{p}}^{\dagger} \coprod_{\hat{\mathfrak{c}}_{\mathfrak{p}, 1}}^{\hat{\mathfrak{p}}_{\mathrm{p}, K}}=\hat{\mathfrak{S}}_{\mathfrak{p}, 1} \hat{\mathfrak{S}}_{\mathfrak{p}}\left(\hat{\mathfrak{S}}_{\mathfrak{p}}^{2}+\vartheta_{N} \mathbb{I}_{\mathscr{H}}\right)^{-1} \coprod_{\hat{\mathfrak{c}}_{\mathfrak{p}, 1}}^{\hat{\mathfrak{c}}_{\mathfrak{p}, K}}
$$

Thereby, $K \in \mathbb{N},\left(\vartheta_{N}\right)_{N \in \mathbb{N}} \subseteq \mathbb{N}$ with $\vartheta_{N} \rightarrow 0, \hat{\mathfrak{c}}_{\mathfrak{p}, 1}, \ldots, \hat{\mathfrak{c}}_{\mathfrak{p}, K}$ are the eigenfunctions of $\hat{\mathfrak{S}}_{\mathfrak{p}}$ associated to the first biggest eigenvalues $\hat{\mathfrak{c}}_{\mathfrak{p}, 1} \geq \cdots \geq \hat{c}_{\mathfrak{p}, K}$ and $\coprod_{\hat{\mathfrak{c}}_{\mathfrak{p}, 1}}^{\hat{\mathfrak{c}}_{\mathfrak{p}}}$ is the operator projecting on $\operatorname{lin}\left\{\hat{\mathfrak{c}}_{\mathfrak{p}, 1}, \ldots, \hat{\mathfrak{c}}_{\mathfrak{p}, K}\right\} \subseteq \mathscr{H}{ }^{\mathfrak{p}}$.

Theorem 3.3. Let Assumptions 3.1-3.2 and let Assumption 3.3 for the eigenvalues of $\mathfrak{S}_{\mathfrak{p}}$ hold. Further, let $\left(\Phi_{\mathfrak{p}, i j}\right)_{i, j \in \mathbb{N}}$ be the CONS of $\mathcal{S}_{\mathscr{H}}, \mathscr{H}$ defined by $\Phi_{\mathfrak{p}, i j}:=\mathfrak{c}_{\mathfrak{p}, i} \otimes \mathfrak{c}_{j}$ for all $i, j$, where $\left(\mathfrak{c}_{\mathfrak{p}, i}\right)_{i \in \mathbb{N}} \subseteq \mathscr{H}^{\mathfrak{p}}$ and $\left(\mathfrak{c}_{j}\right)_{j \in \mathbb{N}} \subseteq \mathscr{H}$ are the eigenfunction sequences of the covariance operators $\mathfrak{S}_{\mathfrak{p}}$ resp. $\mathscr{C}_{0 ; \mathscr{Z}}$.

(a) Let $\mathscr{J}_{\mathfrak{p}, K}:=\left\{\Phi_{\mathfrak{p}, i j} \mid 1 \leq i, j \leq K\right\}$ with $K \in \mathbb{N}$. If $\left\langle\boldsymbol{\alpha}_{[\mathfrak{p}]}\left(\mathfrak{c}_{\mathfrak{p}, l}\right), \mathfrak{c}_{j}\right\rangle_{\mathscr{H}}=0$ for all $j>K, l \leq K$, then

$$
\left\|\hat{\boldsymbol{\alpha}}_{[\mathfrak{p}]}-\coprod_{\mathscr{J}_{\mathfrak{p}, K}} \boldsymbol{\alpha}_{[\mathfrak{p}]}\right\|_{\mathcal{S}_{\mathscr{H}, \mathfrak{H}}}^{2}=\mathrm{O}_{\mathbb{P}}\left(N^{-1}\right)
$$

(b) Let also $\left(\boldsymbol{\alpha}_{[\mathfrak{p}]},\left(\Phi_{\mathfrak{p}, i j}\right)_{i, j}\right)$ satisfy Assumption 3.4 for some $\beta>0$. Then, for any sequence $K=K_{N}=$ $\Xi(1, N)$ with $c_{\mathfrak{p}, K}^{-4} K^{2 \beta+1}=\mathrm{O}(N)$ and $\sum_{l=1}^{K}\left(\frac{c_{\mathfrak{p}, l}^{2}}{c_{\mathfrak{p}, l}^{2}+\vartheta_{N}}\right)^{2} \sum_{j>K}\left\langle\boldsymbol{\alpha}_{[\mathfrak{p}]}\left(\mathfrak{c}_{\mathfrak{p}, l}\right), \mathfrak{c}_{j}\right\rangle_{\mathscr{H}}^{2}=\mathrm{O}\left(K^{-2 \beta}\right)$ holds

$$
\left\|\hat{\boldsymbol{\alpha}}_{[\mathfrak{p}]}-\boldsymbol{\alpha}_{[\mathfrak{p}]}\right\|_{\mathcal{S}_{\mathscr{H}}, \mathscr{H}}^{2}=\mathrm{O}_{\mathbb{P}}\left(K^{-2 \beta}\right) .
$$

Remark 3.2. The assumption $\left\langle\boldsymbol{\alpha}_{[\mathfrak{p}]}\left(\mathfrak{c}_{\mathfrak{p}, l}\right), \mathfrak{c}_{j}\right\rangle_{\mathscr{H}}=0$ for all $j>K, l \leq K$ in Theorem 3.3 (a), which is necessary for technical conversions in the proof, can be interpreted as a slightly milder condition than the condition that the operators $\boldsymbol{\alpha}_{[\mathfrak{p}]}$ and $\mathfrak{S}_{\mathfrak{p}}$ commute. Further, the stated assumption is similar to the assumption used in Turbillon et al. (2007) for the estimation of the operator in a functional MA(1) model.

Example 3.1. Let the assumptions of Theorem 3.3 hold.

(a) Assume $c_{\mathfrak{p}, N} \asymp e^{-N}$ and let $K_{N}:=1+\left\lfloor\frac{\ln (N)}{4+b}\right\rfloor$ for all $N \in \mathbb{N}$ for some $b>0$. Then $K=K_{N}=$ $\Xi(1, N), c_{\mathfrak{p}, K}^{-4} K^{2 \beta+1} \asymp N^{\frac{4}{4+b}} \ln ^{2 \beta+1}(N)=\mathrm{O}(N)$ and consequently according to Theorem 3.3:

$$
\left\|\hat{\boldsymbol{\alpha}}_{[\mathfrak{p}]}-\boldsymbol{\alpha}_{[\mathfrak{p}]}\right\|_{\mathcal{S}_{\mathscr{H}} \mathfrak{p}, \mathscr{H}}^{2}=\mathrm{O}_{\mathbb{P}}\left(\ln ^{-2 \beta}(N)\right) .
$$

(b) Impose $c_{\mathfrak{p}, N} \asymp N^{-a}$ for some $a>1$ and let $K_{N}:=1+\left\lfloor N^{\frac{1}{1+4 a+2 \beta}}\right\rfloor$ for all $N \in \mathbb{N}$. Then $K=K_{N}=$ $\Xi(1, N), c_{\mathfrak{p}, K}^{-4} K^{2 \beta+1} \asymp K^{1+4 a+2 \beta} \asymp N$ and after Theorem 3.3:

$$
\left\|\hat{\boldsymbol{\alpha}}_{[\mathfrak{p}]}-\boldsymbol{\alpha}_{[\mathfrak{p}]}\right\|_{\mathcal{S}_{\mathscr{H} \mathfrak{p}, \mathscr{H}}}^{2}=\mathrm{O}_{\mathbb{P}}\left(N^{-\frac{2 \beta}{1+4 a+2 \beta}}\right) .
$$




\subsection{Operator estimation in the functional GARCH model}

Throughout this section, $\mathscr{X}:=\left(\mathscr{X}_{k}\right)_{k \in \mathbb{Z}}$ is a $\mathscr{H}$-valued $\operatorname{GARCH}(\mathfrak{p}, \mathfrak{q})$ with $\mathfrak{p}, \mathfrak{q} \in \mathbb{N}$ and $\mathscr{Z}:=\left(\mathscr{Z}_{k}\right)_{k \in \mathbb{Z}}=$ $\left(\mathscr{X}_{k}^{2}-m_{2}\right)_{k \in \mathbb{Z}}$ the corresponding $\mathscr{H}$-valued ARMA $(\mathfrak{r}, \mathfrak{q})$ processes with time series of innovations $\boldsymbol{\nu}:=$ $\left(\nu_{k}\right)_{k \in \mathbb{Z}}=\left(\mathscr{X}_{k}^{2}-\sigma_{k}^{2}\right)_{k \in \mathbb{Z}}$ (see p.5). $\mathscr{Z}$ satisfies the following.

Assumption 3.5. $\mathscr{Z}$ is an invertible, linear process w.r.t. $\boldsymbol{\nu}$ with representation as inverted time series

$$
\mathscr{Z}_{k}=\nu_{k}+\sum_{i=1}^{\infty} \pi_{i}\left(\mathscr{Z}_{k-i}\right)
$$

a.s. for all $k$ where $\left(\pi_{i}\right)_{i \in \mathbb{N}} \subseteq \mathcal{S}_{\mathscr{H}}$ with $\sum_{i=1}^{\infty}\left\|\pi_{i}\right\|_{\mathcal{S}_{\mathscr{H}}}<\infty$.

\subsubsection{Derivation of the estimators for the operators in the functional GARCH model}

At first, since $\alpha_{i}:=0_{\mathcal{L}_{\mathscr{C}}}=: \beta_{j}$ for $i>\mathfrak{p}, j>\mathfrak{q}$, the representations (3.2) and (3.35) imply

$$
\mathscr{Z}_{k}=\sum_{i=1}^{\infty}\left(\alpha_{i}+\sum_{j=1}^{i-1 \wedge \mathfrak{q}} \beta_{j} \pi_{i-j}\right)\left(\mathscr{Z}_{k-i}\right)
$$

a.s. for all $k$. Moreover, if Assumptions 3.1-3.2 hold there is no closed subspace $V \subsetneq \mathscr{H}$ with $\mathbb{P}\left(\mathscr{Z}_{0} \in V\right)=1$ which, following from Kühnert (2019), Lemma 4.48 and Remark 4.49, leads to

$$
\pi_{i}=\alpha_{i}+\sum_{j=1}^{i-1 \wedge \mathfrak{q}} \beta_{j} \pi_{i-j}, \quad i \in \mathbb{N} .
$$

Since $\alpha_{i}=0_{\mathcal{L}_{\mathscr{H}}}$ for $i>\mathfrak{p},(3.36)$ implies with $\mathfrak{s}=\mathfrak{p}+\mathfrak{q}$ :

$$
\pi_{\mathfrak{s}}=\sum_{j=1}^{\mathfrak{q}} \beta_{j} \pi_{\mathfrak{s}-j}=\left[\beta_{1} \beta_{2} \cdots \beta_{\mathfrak{q}}\right]\left[\pi_{\mathfrak{s}-1} \pi_{\mathfrak{s}-2} \cdots \pi_{\mathfrak{p}}\right]^{T}=: \boldsymbol{\beta}_{[\mathfrak{q}]} \boldsymbol{\pi}_{[\mathfrak{p}, \mathfrak{q}]}^{T}
$$

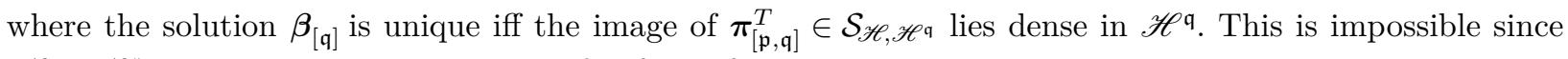
$\mathscr{H} \subsetneq \mathscr{H}^{\mathfrak{q}}$, why we establish estimators for $\beta_{1}, \ldots, \beta_{\mathfrak{q}}$ based on the equation

$$
\boldsymbol{\pi}_{[\mathfrak{s}, \mathfrak{q}]}=\boldsymbol{\beta}_{[\mathfrak{q}]} \prod_{[\mathfrak{s , q}]}: \Longleftrightarrow\left[\begin{array}{c}
\pi_{\mathfrak{s}+\mathfrak{q}-1} \\
\pi_{\mathfrak{s}+\mathfrak{q}-2} \\
\vdots \\
\pi_{\mathfrak{s}}
\end{array}\right]=\left[\beta_{1} \beta_{2} \cdots \beta_{\mathfrak{q}}\right]\left[\begin{array}{cccc}
\pi_{\mathfrak{s}+\mathfrak{q}-2} & \pi_{\mathfrak{s}+\mathfrak{q}-3} & \cdots & \pi_{\mathfrak{s}-1} \\
\pi_{\mathfrak{s}+\mathfrak{q}-3} & \pi_{\mathfrak{s}+\mathfrak{q}-4} & \cdots & \pi_{\mathfrak{s}-2} \\
\vdots & \vdots & \cdots & \vdots \\
\pi_{\mathfrak{s}-1} & \pi_{\mathfrak{s}-2} & \cdots & \pi_{\mathfrak{p}}
\end{array}\right]
$$

The following example illustrates that the image of $\prod_{[\mathfrak{s}, \mathfrak{q}]} \in \mathcal{S}_{\mathscr{H}^{\mathfrak{q}}}$ can lie dense.

Example 3.2. Let $\alpha_{i}:=0_{\mathcal{L}_{\mathscr{C}}}=: \beta_{j}$ for $i \neq \mathfrak{p}, j \neq \mathfrak{q}$ and let $\alpha_{\mathfrak{p}}=\beta_{\mathfrak{q}}=: \gamma$ where $\gamma \in \mathcal{S}_{\mathscr{H}}$ is an operator with dense image satisying $\gamma \neq 0_{\mathcal{L}_{\mathscr{H}}}$ and $\|\gamma\|_{\mathcal{S}_{\mathscr{H}}}<1$. Then, because (3.36) implies $\pi_{i}=\gamma^{k}$ for all $i=\mathfrak{p}+(k-1) \mathfrak{q}$ for some $k \in \mathbb{N}$ and $\pi_{i}=0_{\mathcal{L}_{\mathscr{H}}}$ otherwise, we obtain

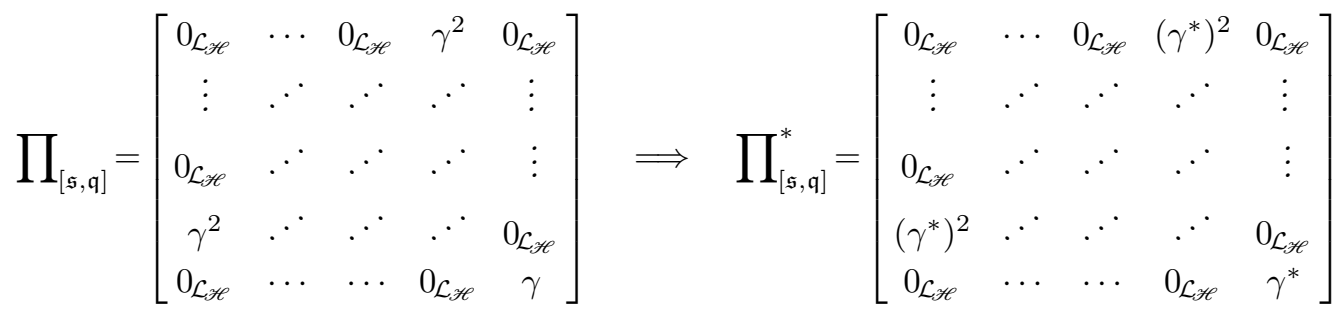

Since the operators $\gamma^{*},\left(\gamma^{*}\right)^{2}$ and hence $\prod_{[\mathfrak{s}, \mathfrak{q}]}^{*}$ are injective, the image of $\prod_{[\mathfrak{s}, \mathfrak{q}]}$ lies dense. 
Due to (3.37), analogously to (3.32), we use

$$
\hat{\boldsymbol{\beta}}_{[\mathfrak{q}]}:=\hat{\boldsymbol{\pi}}_{[\mathfrak{s}, \mathfrak{q}]} \hat{\prod}_{[\mathfrak{s}, \mathfrak{q}]}^{\dagger} \coprod_{\hat{\mathfrak{g}}_{\mathfrak{s}, \mathfrak{q} ; 1}}^{\hat{\mathfrak{g}}_{\mathfrak{s}, \mathfrak{q} ; M}}=\hat{\boldsymbol{\pi}}_{[\mathfrak{s}, \mathfrak{q}]} \hat{\prod}_{[\mathfrak{s}, \mathfrak{q}]}^{*}\left(\hat{\prod}_{[\mathfrak{s}, \mathfrak{q}]} \hat{\prod}_{[\mathfrak{s}, \mathfrak{q}]}^{*}+\theta_{N} \mathbb{I}_{\mathscr{H} \mathfrak{q}}\right)^{-1} \coprod_{\mathfrak{\mathfrak { g }}_{\mathfrak{s}, \mathfrak{q} ; 1}}^{\hat{\mathfrak{g}}_{\mathfrak{s}, \mathfrak{q} ; M}}
$$

to estimate $\boldsymbol{\beta}_{[\mathfrak{q}]}$. Thereby,

$$
\hat{\pi}_{[\mathfrak{s}, \mathfrak{q}]}:=\left[\hat{\pi}_{\mathfrak{s}+\mathfrak{q}-1} \hat{\pi}_{\mathfrak{s}+\mathfrak{q}-2} \cdots \hat{\pi}_{\mathfrak{s}}\right]
$$

is an element of $\mathcal{S}_{\mathscr{H} \mathfrak{q}, \mathscr{H}}$ and

$$
\hat{\prod}_{[\mathfrak{s}, \mathfrak{q}]}:=\left[\begin{array}{cccc}
\hat{\pi}_{\mathfrak{s}+\mathfrak{q}-2} & \hat{\pi}_{\mathfrak{s}+\mathfrak{q}-3} & \cdots & \hat{\pi}_{\mathfrak{s}-1} \\
\hat{\pi}_{\mathfrak{s}+\mathfrak{q}-3} & \hat{\pi}_{\mathfrak{s}+\mathfrak{q}-4} & \cdots & \hat{\pi}_{\mathfrak{s}-2} \\
\vdots & \vdots & \ddots & \vdots \\
\hat{\pi}_{\mathfrak{s}-1} & \hat{\pi}_{\mathfrak{s}-2} & \cdots & \hat{\pi}_{\mathfrak{p}}
\end{array}\right]
$$

is an element of $\mathcal{S}_{\mathscr{H}}$ q. Further, $\hat{\pi}_{k}$ where $k \in \mathbb{N}$, with what we estimate $\pi_{k}$, stands for the $k$-th component of

$$
\hat{\pi}_{L, K}:=\hat{\mathfrak{S}}_{L, K} \hat{\mathfrak{S}}_{L}^{\dagger} \coprod_{\hat{\mathfrak{c}}_{L, 1}}^{\hat{\mathfrak{c}}_{L, K}}=\hat{\mathfrak{S}}_{L, 1} \hat{\mathfrak{S}}_{L}\left(\hat{\mathfrak{S}}_{L}^{2}+\vartheta_{N} \mathbb{I}_{\mathscr{H}^{L}}\right)^{-1} \coprod_{\hat{\mathfrak{c}}_{L, 1}}^{\hat{\mathfrak{c}}_{L, K}}
$$

where $\left(K_{N}\right)_{N \in \mathbb{N}} \subseteq \mathbb{N},\left(L_{N}\right)_{N \in \mathbb{N}} \subseteq \mathbb{N}$ and $\left(\vartheta_{N}\right)_{N \in \mathbb{N}} \subseteq(0, \infty)$ are sequences with $K=K_{N} \rightarrow \infty, L=L_{N} \rightarrow \infty$ resp. $\vartheta_{N} \rightarrow 0$ and where $\hat{\mathfrak{c}}_{L, 1}, \ldots, \hat{\mathfrak{c}}_{L, K}$ are the eigenfunctions of $\hat{\mathfrak{S}}_{L}$ associated to the first biggest eigenvalues $\hat{c}_{L, 1} \geq \cdots \geq \hat{c}_{L, K}$. Further, in $(3.38),\left(M_{N}\right)_{N \in \mathbb{N}} \subseteq \mathbb{N}$ and $\left(\theta_{N}\right)_{N \in \mathbb{N}} \subseteq(0, \infty)$ are sequences with $M=M_{N} \rightarrow \infty$ resp. $\theta_{N} \rightarrow 0$ and $\left(\hat{\mathfrak{g}}_{\mathfrak{s}, \mathfrak{q} ; j}\right)_{j \in \mathbb{N}}$ is the eigenfunction sequence of $\hat{\prod}_{[\mathfrak{s}, \mathfrak{q}]} \hat{\prod}_{[\mathfrak{s}, \mathfrak{q}]}^{*} \in \mathcal{S}_{\mathscr{H} \mathfrak{q}}$ with associated eigenvalue sequence $\left(\hat{g}_{\mathfrak{s}, \mathfrak{q} ; j}\right)_{j \in \mathbb{N}}$ which decreases monotonically w.l.o.g. Because of $(3.36)$, it is hence plausible to use

$$
\hat{\alpha}_{i}:=\hat{\pi}_{i}-\sum_{j=1}^{i-1 \wedge \mathfrak{q}} \hat{\beta}_{j} \hat{\pi}_{i-j}
$$

as an estimator for $\alpha_{i}$ with $i=1, \ldots, \mathfrak{p}$ where $\hat{\beta}_{j}$ is the $j$-th component of $\hat{\boldsymbol{\beta}}_{[\mathfrak{q}]}$ and where $\hat{\alpha}_{1}:=\hat{\pi}_{1}$.

\subsubsection{Upper bounds of the estimation errors for the operators $\pi_{i}$}

The following Theorem is crucial for the derivation of asymptotic upper bounds of the estimation errors of the $\mathrm{ARCH}$ and GARCH operators.

Theorem 3.4. Let Assumptions 3.1-3.2 and 3.5 hold. Let $\beta>0, L=L_{N}=\Xi(1, N), K=K_{N}=\Xi\left(1, \sqrt[3]{L^{-1} N}\right)$ and $\vartheta_{N}=\mathrm{O}\left(c_{L, K}^{-2} K^{-\beta}\right)$. Thereby, $\left(c_{L, j}\right)_{j}$ is the eigenvalue sequence of $\mathfrak{S}_{L}$ satisfying $c_{L, K}=\Omega\left(\sqrt{L^{3} N^{-1}}\right)$, $\sum_{l=1}^{K}\left(\frac{c_{L, l}^{2}}{c_{L, l}^{2}+\vartheta_{N}}\right)^{2} \sum_{j>K}\left\langle\boldsymbol{\pi}_{L}\left(\mathfrak{c}_{L, l}\right), \mathfrak{c}_{j}\right\rangle_{\mathscr{H}}^{2}=\mathrm{O}\left(K^{-2 \beta}\right)$ and $c_{L, K}^{-1} \sqrt{K L^{3} N^{-1}}=\mathrm{O}\left(K^{-(2 \beta+1)}\right)$ if $\sum_{l>L}\left\|\pi_{l}\right\|_{\mathcal{L}_{\mathscr{H}}}=$ $\mathrm{O}\left(c_{L, K}^{-1} \sqrt{K L^{3} N^{-1}}\right)$ resp. $L^{-2} N\left(\sum_{l>L}\left\|\pi_{l}\right\|_{\mathcal{L}_{\mathscr{H}}}\right)^{4}=\mathrm{O}\left(K^{1-2 \beta}\right)$ if $c_{L, K}^{-1} \sqrt{K L^{3} N^{-1}}=\mathrm{O}\left(\sum_{l>L}\left\|\pi_{l}\right\|_{\mathcal{L}_{\mathscr{H}}}\right)$. At last, for all $L$, let Assumption 3.3 hold for the eigenvalue sequence $\left(c_{L, j}\right)_{j}$ and let $\left(\boldsymbol{\pi}_{L},\left(\Phi_{L, i j}\right)_{i, j}\right)$ satisfy Assumption 3.4 for $\beta$, see Theorem 3.3. Then, for all $i \in \mathbb{N}$ :

$$
\left\|\hat{\pi}_{i}-\pi_{i}\right\|_{\mathcal{S}_{\mathscr{H}}}^{2}=\mathrm{O}_{\mathbb{P}}\left(K^{-2 \beta}\right)
$$

\subsubsection{Upper bounds of the estimation errors for the GARCH operators}

Here, we need

$$
\left\|\hat{\boldsymbol{\pi}}_{[\mathfrak{s}, \mathfrak{q}]}-\boldsymbol{\pi}_{[\mathfrak{s}, \mathfrak{q}]}\right\|_{\mathcal{S}_{\mathscr{H} \mathfrak{p}, \mathscr{H}}}^{2}=\sum_{i=0}^{\mathfrak{q}-1}\left\|\hat{\pi}_{\mathfrak{s}+i}-\pi_{\mathfrak{s}+i}\right\|_{\mathcal{S}_{\mathscr{H}}}^{2}=\mathrm{O}_{\mathbb{P}}\left(K^{-2 \beta}\right)
$$


as well as

$$
\begin{aligned}
\left\|\hat{\prod}_{[\mathfrak{s , q}]} \hat{\prod}_{[\mathfrak{s , q}]}^{*}-\prod_{[\mathfrak{s}, \mathfrak{q}]} \prod_{[\mathfrak{s , \mathfrak { q } ]}]}^{*}\right\|_{\mathcal{S}_{\mathscr{H} \mathfrak{q}}}^{2} & =\sum_{i, j=0}^{\mathfrak{q}-1}\left\|\sum_{k=\mathfrak{p}}^{\mathfrak{s}-1} \hat{\pi}_{k+i} \hat{\pi}_{k+j}^{*}-\pi_{k+i} \pi_{k+j}^{*}\right\|_{\mathcal{S}_{\mathscr{H}}}^{2} \\
& \precsim \sum_{i, j=0}^{\mathfrak{q}-1} \sum_{k=\mathfrak{p}}^{\mathfrak{s}-1}\left\|\hat{\pi}_{k+i}\right\|_{\mathcal{S}_{\mathscr{H}}}^{2}\left\|\hat{\pi}_{k+j}^{*}-\pi_{k+j}^{*}\right\|_{\mathcal{S}_{\mathscr{H}}}^{2}+\left\|\hat{\pi}_{k+i}-\pi_{k+i}\right\|_{\mathcal{S}_{\mathscr{H}}}^{2}\left\|\pi_{k+j}^{*}\right\|_{\mathcal{S}_{\mathscr{H}}}^{2} \\
& =\mathrm{O}_{\mathbb{P}}\left(K^{-2 \beta}\right)
\end{aligned}
$$

which is both true after (3.43). According to Corollary 3.1, the identities (3.13) and (3.45) imply

$$
\sup _{j \in \mathbb{N}}\left(\hat{g}_{\mathfrak{s}, \mathfrak{q} ; j}-g_{\mathfrak{s}, \mathfrak{q} ; j}\right)^{2}=\mathrm{O}\left(K^{-2 \beta}\right)
$$

where $\left(g_{\mathfrak{s , q} ; j}\right)_{j \in \mathbb{N}}$ is the w.l.o.g. monotonically decreasing eigenvalue sequence associated to the eigenfunction sequence $\left(\mathfrak{g}_{\mathfrak{s}, \mathfrak{q} ; j}\right)_{j \in \mathbb{N}}$ of $\prod_{[\mathfrak{s}, \mathfrak{q}]} \prod_{[\mathfrak{s , q}]}^{*} \in \mathcal{S}_{\mathscr{H} \mathfrak{q}}$. Moreover, if $g_{\mathfrak{s}, \mathfrak{q} ; M}=\Xi\left(K^{-\beta}, 1\right)$ with $M=M_{N}=\Xi(1, N)$, then

$$
\hat{g}_{\mathfrak{s}, \mathfrak{q} ; M}=\mathrm{O}_{\mathbb{P}}\left(g_{\mathfrak{s}, \mathfrak{q} ; M}\right) \quad \text { and } \quad g_{\mathfrak{s}, \mathfrak{q} ; M}=\mathrm{O}_{\mathbb{P}}\left(\hat{g}_{\mathfrak{s}, \mathfrak{q} ; M}\right)
$$

after Corollary 3.1 and if also $\prod_{[\mathfrak{s , q}]} \prod_{[\mathfrak{s , q}]}^{*}$ is injective and satisfies Assumption 3.3, Lemma 3.3 yields

$$
\sup _{j \leq M}\left\|\hat{\mathfrak{g}}_{\mathfrak{s , q} ; j ;}^{\prime \prime \prime}-\mathfrak{g}_{\mathfrak{s}, \mathfrak{q} ; j}\right\|_{\mathscr{H} \mathfrak{q}}^{2}=\mathrm{O}_{\mathbb{P}}\left(\mathfrak{g}_{\mathfrak{s}, \mathfrak{q} ; M}^{-2} K^{-2 \beta}\right) .
$$

Theorem 3.5. Let the assumptions of Theorem 3.4 hold. Let $\prod_{[\mathfrak{s , q}, \mathfrak{[ s , q ]}} \prod_{[\mathfrak{s}]}^{*}$ be injective and let its eigenvalue sequence $\left(g_{\mathfrak{s}, \mathfrak{q} ; j}\right)_{j}$ satisfy Assumption 3.3. Also, let $M=M_{N}=\Xi(1, N), \theta_{N}=\mathrm{O}\left(K^{-\beta / 2}\right), g_{\mathfrak{s , q} ; M^{-2}}^{-2} M^{\beta}=\mathrm{O}\left(K^{\beta}\right)$ as well as $\sum_{l=1}^{M}\left(\frac{g_{\mathfrak{s q}, l}^{2}}{g_{\mathfrak{s}, q ;}^{2}+\theta_{N}}\right)^{2} \sum_{j>M}\left\langle\boldsymbol{\beta}_{[\mathfrak{q}]}\left(\mathfrak{g}_{\mathfrak{s}, \mathfrak{q} ; l}\right), \mathfrak{c}_{j}\right\rangle_{\mathscr{H}}^{2}=\mathrm{O}\left(M^{-2 \beta}\right)$ hold, and let $\left(\boldsymbol{\beta}_{[\mathfrak{q}]},\left(\Phi_{\mathfrak{s , q} ; i j}\right)_{i, j}\right)$ satisfy Assumption 3.4 for $\beta$ where $\Phi_{\mathfrak{s}, \mathfrak{q} ; i j}:=\mathfrak{g}_{\mathfrak{s , q} ; j} \otimes \mathfrak{c}_{j}$. Then,

$$
\left\|\hat{\alpha}_{i}-\alpha_{i}\right\|_{\mathcal{S}_{\mathscr{H}}}^{2}= \begin{cases}\mathrm{O}_{\mathbb{P}}\left(K^{-2 \beta}\right), & i=1 \\ \mathrm{O}_{\mathbb{P}}\left(M^{-2 \beta}\right), & i=2, \ldots, \mathfrak{p}\end{cases}
$$

and for all $j=1, \ldots, \mathfrak{q}$ :

$$
\left\|\hat{\beta}_{j}-\beta_{j}\right\|_{\mathcal{S}_{\mathscr{H}}}^{2}=\mathrm{O}_{\mathbb{P}}\left(M^{-2 \beta}\right)
$$

\section{Conclusions}

This paper provides sufficient conditions for the existence of strictly stationary solutions, weak dependence and finite moments of $\operatorname{ARCH}(\mathfrak{p})$ and $\operatorname{GARCH}(\mathfrak{p}, \mathfrak{q})$ processes for any order $\mathfrak{p}, \mathfrak{q} \in \mathbb{N}$ with values in $L^{p}[0,1]$ with $p \in[1, \infty), C[0,1]$ and other function spaces with domain $[0,1]$. For any order, in $L^{2}[0,1]$, explicit asymptotic upper bounds of the estimation errors for the shift term and the complete operators of ARCH and GARCH processes and the projections of the ARCH operators on a finite-dimensional subspace are derived. The operators are estimated by Yule-Walker estimators. The estimation of the GARCH operators also involves the estimation of operators in invertible, linear processes represented as inverted time series.

The probabilistic features of our ARCH and GARCH processes are studied in Section 2. Theorem 2.1 and Lemma 2.1 present sufficient conditions for the existence of strictly stationary solutions resp. for weak dependence and finite moments for our $\mathrm{ARCH}$ and GARCH processes for any order. Theorem 2.1 generalizes Cerovecki et al. (2019), Theorem 1 under a milder condition, Hörmann et al. (2013), Theorem 2.1 and 2.3 and Aue et al. (2017), Theorem 2.1 and 2.2. To the best of our knowledge, for functional ARCH(p) resp. $\operatorname{GARCH}(\mathfrak{p}, \mathfrak{q})$ processes with $\mathfrak{p}>1$ resp. $\mathfrak{r}=\max (\mathfrak{p}, \mathfrak{q})>1$, a moment condition as (2.10) in Theorem 2.1 and Lemma 2.1 are new. The parameters of $L^{2}[0,1]$-valued ARCH and GARCH processes are estimated in Section 3. In order to derive asymptotic upper bounds of the estimaton errors for the ARCH and GARCH 
parameters, convergence results stating asymptotic upper bounds of the estimation errors for certain means, covariance as well as lag- $h$-covariance operators, eigenfunctions and eigenvalues are presented which are also useful beyond the context of ARCH and GARCH. Theorems 3.2-3.5 present the main results. Theorem 3.2 states explicit asymptotic upper bounds for the estimation errors for the shift term in ARCH and GARCH. For the ARCH processes, Theorem 3.3 provides explicit asymptotic upper bounds for the estimation errors for the projections of the operators on finite-dimensional subspaces in part (a), and the complete operators in part (b). A similar result as (a) for $\mathfrak{p}=1$ was already stated in Hörmann et al. (2013) by imposing an integral operator and estimating its kernel. As far as we know, (a) with $\mathfrak{p}>1$ and (b) are new. Theorem 3.4 is a convergence result stating explicit asymptotic upper bounds for the operators of invertible, linear processes represented as inverted time series from which one immediately obtains a result with the same upper bounds for the operators in the associated linear process, see Kühnert (2019), Section 4.4.1.3. Both results are valid without the context of ARCH and GARCH. They extend some results in Aue \& Klepsch (2017) and Klepsch \& Klüppelberg (2017) where a different approach was made. At last, Theorem 3.5 provides explicit asymptotic upper bounds for the estimation errors for the complete GARCH operators. Projections of these operators on finite-dimensional subspaces were already estimated in Aue et al. (2017) for $\mathfrak{p}=1=\mathfrak{q}$ by least squares estimators and in Cerovecki et al. (2019) for any order by a quasi-likelihood approach. By the same approach, Cerovecki et al. (2019) also estimated the complete operators in the case $\mathfrak{p}=1=\mathfrak{q}$. To the best of our knowledge, estimating the complete operators in Theorem 3.5 for $\max (\mathfrak{p}, \mathfrak{q})>1$ is new and explicit asymptotic upper bounds of estimation errors for complete operators of ARCH, ARMA, GARCH, invertible and linear processes as in the Theorems 3.3-3.5 have not been derived before.

We leave the investigations concerning the probabilistic properties of ARCH and GARCH processes in general, separable Banach spaces behind for future research. An open problem is also the estimation of the orders of functional ARCH and GARCH processes, see Kokoszka \& Reimherr (2013). Concerning parameter estimation in functional ARCH and GARCH processes, open problems are the estimation in general, separable Banach spaces, see Ruiz-Medina \& Álvarez-Liébana (2019), the asymptotic distribution of the estimations errors when estimating the parameters without projecting them on a finite-dimensional subspace, see Aue et al. (2017) and Cerovecki et al. (2019) for the parameters projected on a finite-dimensinal subspace, and the asymptotic lower bounds for the estimations errors.

\section{Proofs}

In various conversions, we utilize the inequality

$$
\left(\sum_{k=1}^{n} a_{k}\right)^{\nu} \leq \begin{cases}\sum_{k=1}^{n} a_{k}^{\nu}, & \nu \in(0,1] \\ n^{\nu-1} \sum_{k=1}^{n} a_{k}^{\nu}, & \nu \in(1, \infty)\end{cases}
$$

for $n \in \mathbb{N}$ and $a_{1}, \ldots, a_{n} \geq 0$, thus $\left(\sum_{k=1}^{n} a_{k}\right)^{\nu} \precsim \sum_{k=1}^{n} a_{k}^{\nu}$, and the operator valued Hölder's inequality which we state below. Let $\left(\mathcal{H},\langle\cdot, \cdot\rangle_{\mathcal{H}}\right),\left(\mathcal{H}^{\prime},\langle\cdot, \cdot\rangle_{\mathcal{H}^{\prime}}\right)$ and $\left(\mathcal{H}^{\prime \prime},\langle\cdot, \cdot\rangle_{\mathcal{H}^{\prime \prime}}\right)$ be Hilbert spaces. For $p \in[1, \infty], \mathscr{S}_{\mathcal{H}, \mathcal{H}^{\prime}}^{p} \subseteq$ $\mathcal{L}_{\mathcal{H}, \mathcal{H}^{\prime}}$ denotes the $p$-th Schatten-class where $\mathscr{S}_{\mathcal{H}, \mathcal{H}^{\prime}}^{1}=\mathcal{N}_{\mathcal{H}, \mathcal{H}^{\prime}}, \mathscr{S}_{\mathcal{H}, \mathcal{H}^{\prime}}^{2}=\mathcal{S}_{\mathcal{H}, \mathcal{H}^{\prime}}$ and $\mathscr{S}_{\mathcal{H}, \mathcal{H}^{\prime}}^{\infty}:=\mathcal{L}_{\mathcal{H}, \mathcal{H}^{\prime}}$. For any $p \in[1, \infty), A \in \mathscr{S}_{\mathcal{H}, \mathcal{H}^{\prime}}^{p}$ if $\sum_{j=1}^{\infty} s_{j}^{p}(A)<\infty$, where $s_{j}(A)$ is the $j$-th singular value of $A \in \mathcal{L}_{\mathcal{H}, \mathcal{H}^{\prime}}$, and $\mathscr{S}_{\mathcal{H}, \mathcal{H}^{\prime}}^{p}$ is endowed with the norm $\|\cdot\|_{\mathscr{S}_{\mathcal{H}, \mathcal{H}^{\prime}}^{p}}:=\left(\sum_{j=1}^{\infty} s_{j}^{p}(\cdot)\right)^{1 / p}$. Now, let $p, q, r \in[1, \infty]$ with $\frac{1}{p}+\frac{1}{q}=\frac{1}{r}$ where $\frac{1}{\infty}:=0$ and let $A \in \mathscr{S}_{\mathcal{H}^{\prime}, \mathcal{H}^{\prime \prime}}^{q}$ and $B \in \mathscr{S}_{\mathcal{H}, \mathcal{H}^{\prime}}^{p}$. Then, after the operator valued Hölder's inequality (see Kaballo (2014), Theorem 11.2), $A B \in \mathscr{S}_{\mathcal{H}, \mathcal{H}^{\prime \prime}}^{r}$ with

$$
\|A B\|_{\mathscr{S}_{\mathcal{H}, \mathcal{H}^{\prime \prime}}^{r}} \leq 2^{1 / r}\|A\|_{\mathscr{S}_{\mathcal{H}^{\prime}, \mathcal{H}^{\prime \prime}}^{q}}\|B\|_{\mathscr{S}_{\mathcal{H}, \mathcal{H}^{\prime}}^{p}}
$$

Proof of Theorem 2.1. (a) The state-space form (2.2) yields

$$
\boldsymbol{\varsigma}_{k}^{(\mathfrak{p}, \mathfrak{q})}=\boldsymbol{\delta}_{k}^{(\mathfrak{p}, \mathfrak{q})}+\sum_{m=1}^{\infty} \boldsymbol{\Psi}_{k}^{(\mathfrak{p}, \mathfrak{q})} \Psi_{k-1}^{(\mathfrak{p}, \mathfrak{q})} \cdots \boldsymbol{\Psi}_{k-m+1}^{(\mathfrak{p}, \mathfrak{q})}\left(\boldsymbol{\delta}_{k-m}^{(\mathfrak{p}, \mathfrak{q})}\right)
$$


a.s. for all $k$ if the series converges a.s. Further, (2.7) implies

$$
\varlimsup_{m \rightarrow \infty} \frac{1}{m} \ln \left\|\mathbf{\Psi}_{k}^{(\mathfrak{p}, \mathfrak{q})} \mathbf{\Psi}_{k-1}^{(\mathfrak{p}, \mathfrak{q})} \cdots \boldsymbol{\Psi}_{k-m+1}^{(\mathfrak{p}, \mathfrak{q})}\left(\boldsymbol{\delta}_{k-m}^{(\mathfrak{p}, \mathfrak{q})}\right)\right\|_{F^{\mathfrak{s}}} \leq \gamma^{(\mathfrak{p}, \mathfrak{q})}+\varlimsup_{m \rightarrow \infty} \frac{1}{m} \ln \left\|\boldsymbol{\delta}_{k-m}^{(\mathfrak{p}, \mathfrak{q})}\right\|_{F^{\mathfrak{s}}}
$$

By definition of $\boldsymbol{\delta}_{k-m}^{(\mathfrak{p}, \mathfrak{q})}$ and due to $0<\|\delta\|_{F} \leq\|\delta\|_{\dot{F}},\left\|\boldsymbol{\delta}_{k-m}^{(\mathfrak{p}, \mathfrak{q})}\right\|_{F^{\mathfrak{s}}}^{2} \leq\|\delta\|_{\dot{F}}^{2}\left(1+\left\|\varepsilon_{k-m}^{2}\right\|_{\dot{F}}^{2}\right)$ which leads because of (2.3) to $\mathbb{E} \ln ^{+}\left\|\boldsymbol{\delta}_{k-m}^{(\mathfrak{p}, \mathfrak{q})}\right\|_{F^{\mathfrak{s}}}<\infty$. Since $\gamma^{(\mathfrak{p}, \mathfrak{q})}<0$

$$
\varlimsup_{m \rightarrow \infty}\left\|\Psi_{k}^{(\mathfrak{p}, \mathfrak{q})} \mathbf{\Psi}_{k-1}^{(\mathfrak{p}, \mathfrak{q})} \cdots \mathbf{\Psi}_{k-m+1}^{(\mathfrak{p}, \mathfrak{q})}\left(\boldsymbol{\delta}_{k-m}^{(\mathfrak{p}, \mathfrak{q})}\right)\right\|_{F^{\mathfrak{s}}}^{1 / m}=e^{\gamma^{(\mathfrak{p}, \mathfrak{q})}}<1
$$

a.s. and thus the series in (5.2) converges a.s. Hence, there exists a unique solution $\left(\boldsymbol{\varsigma}_{k}^{(\mathfrak{p}, \mathfrak{q})}\right)_{k}$ of $(2.2)($ for uniqueness see Cerovecki et al. (2019), p.19) and thus also of (2.1). By definition of $\boldsymbol{\varsigma}_{k}^{(\mathfrak{p}, \mathfrak{q})}$ and due to (5.2), $\sigma_{k}^{2}=f\left(\varepsilon_{k-1}, \varepsilon_{k-2}, \ldots\right)$ a.s. for all $k$ for some measurable function $f: F^{\infty} \rightarrow F$, thus $\left(\sigma_{k}^{2}\right)_{k}$ and $\left(\mathscr{X}_{k}\right)_{k}$ are nonanticipative w.r.t. $\left(\varepsilon_{k}\right)_{k}$ and strictly stationary as well as ergodic after Stout (1974), Theorem 3.5.8.

(b) $\nu>0, \psi_{n, \nu}^{(\mathfrak{p}, \mathfrak{q})}<1,(2.10)$, sub-multiplicativity of $\|\cdot\|_{\mathcal{L}_{F^{\mathfrak{s}}}}$ and Jensen's inequality imply

$$
\gamma^{(\mathfrak{p}, \mathfrak{q})} \leq \lim _{m \rightarrow \infty} \frac{1}{m n} \mathbb{E} \ln \left(\prod_{l=1}^{m}\left\|\Psi_{l n}^{(\mathfrak{p}, \mathfrak{q})} \mathbf{\Psi}_{l n-1}^{(\mathfrak{p}, \mathfrak{q})} \ldots \boldsymbol{\Psi}_{(l-1) n+1}^{(\mathfrak{p}, \mathfrak{q})}\right\|_{\mathcal{L}_{F^{\mathfrak{s}}}}\right) \leq \frac{1}{n \nu} \ln \left(\psi_{n, \nu}^{(\mathfrak{p}, \mathfrak{q})}\right)<0
$$

Proof of Corollary 2.1. Assume $N=m n$ for some $m \in \mathbb{N}$ w.l.o.g. Then, since $\psi_{n, \nu}^{(\mathfrak{p}, \mathfrak{q})}<1$ and $\left(\mathbf{\Psi}_{k}^{(\mathfrak{p}, \mathfrak{q})}\right)_{k \in \mathbb{Z}}$ is i.i.d., the assertion follows from

$$
\begin{aligned}
\mathbb{E}\left\|\boldsymbol{\varsigma}_{N}^{(\mathfrak{p}, \mathfrak{q})}-\tilde{\boldsymbol{\varsigma}}_{N}^{(\mathfrak{p}, \mathfrak{q})}\right\|_{F^{\mathfrak{s}}}^{\nu} & =\mathbb{E}\left\|\boldsymbol{\Psi}_{N}^{(\mathfrak{p}, \mathfrak{q})} \mathbf{\Psi}_{N-1}^{(\mathfrak{p}, \mathfrak{q})} \cdots \boldsymbol{\Psi}_{1}^{(\mathfrak{p}, \mathfrak{q})}\left(\boldsymbol{\varsigma}_{0}^{(\mathfrak{p}, \mathfrak{q})}-\tilde{\boldsymbol{\varsigma}}_{0}^{(\mathfrak{p}, \mathfrak{q})}\right)\right\|_{F^{\mathfrak{s}}}^{\nu} \\
& \leq\left(\psi_{n, \nu}^{(\mathfrak{p}, \mathfrak{q})}\right)^{N / n} \mathbb{E}\left\|\boldsymbol{\varsigma}_{0}^{(\mathfrak{p}, \mathfrak{q})}-\tilde{\boldsymbol{\varsigma}}_{0}^{(\mathfrak{p}, \mathfrak{q})}\right\|_{F^{\mathfrak{s}}}^{\nu}
\end{aligned}
$$

Proof of Lemma 2.1. (a) For all $\nu>0$, we have

$$
\begin{aligned}
\left\|\boldsymbol{\varsigma}_{0}^{(\mathfrak{p}, \mathfrak{q})}\right\|_{F^{\mathfrak{s}}}^{\nu} & \precsim\left\|\boldsymbol{\delta}_{0}^{(\mathfrak{p}, \mathfrak{q})}\right\|_{F^{\mathfrak{s}}}^{\nu}+\left(\sum_{m=1}^{\infty}\left\|\boldsymbol{\Psi}_{0}^{(\mathfrak{p}, \mathfrak{q})} \mathbf{\Psi}_{-1}^{(\mathfrak{p}, \mathfrak{q})} \ldots \mathbf{\Psi}_{-m+1}^{(\mathfrak{p}, \mathfrak{q})}\right\|_{\mathcal{L}_{F^{\mathfrak{s}}}}\left\|\boldsymbol{\delta}_{-m}^{(\mathfrak{p}, \mathfrak{q})}\right\|_{F^{\mathfrak{s}}}\right)^{\nu} \\
& \precsim\left\|\boldsymbol{\delta}_{0}^{(\mathfrak{p}, \mathfrak{q})}\right\|_{F^{\mathfrak{s}}}^{\nu}+\sum_{m=1}^{n-1}\left\|\boldsymbol{\delta}_{-m}^{(\mathfrak{p}, \mathfrak{q})}\right\|_{F^{\mathfrak{s}}}^{\nu} \prod_{l=1}^{m}\left\|\boldsymbol{\Psi}_{-m+1}^{(\mathfrak{p}, \mathfrak{q})}\right\|_{\mathcal{L}_{F^{\mathfrak{s}}}}^{\nu}+\left(\sum_{m=n}^{\infty}\left\|\boldsymbol{\Psi}_{0}^{(\mathfrak{p}, \mathfrak{q})} \boldsymbol{\Psi}_{-1}^{(\mathfrak{p}, \mathfrak{q})} \ldots \boldsymbol{\Psi}_{-m+1}^{(\mathfrak{p}, \mathfrak{q})}\right\|_{\mathcal{L}_{F^{\mathfrak{s}}}}\left\|\boldsymbol{\delta}_{-m}^{(\mathfrak{p}, \mathfrak{q})}\right\|_{F^{\mathfrak{s}}}\right)^{\nu} .
\end{aligned}
$$

Moreover, $\mathbb{E}\left\|\varepsilon_{0}^{2}\right\|_{\dot{F}}^{\nu}<\infty$ implies $\mathbb{E}\left\|\boldsymbol{\delta}_{0}^{(\mathfrak{p}, \mathfrak{q})}\right\|_{F^{\mathfrak{s}}}^{\nu}<\infty$ as well as $\mathbb{E}\left\|\mathbf{\Psi}_{0}^{(\mathfrak{p}, \mathfrak{q})}\right\|_{\mathcal{L}_{F^{\mathfrak{s}}}}^{\nu}<\infty$. From the definition of $\mathbf{\Psi}_{k}^{(\mathfrak{p}, \mathfrak{q})}$ and $\boldsymbol{\delta}_{k}^{(\mathfrak{p}, \mathfrak{q})}$ for all $k$ and since $\left(\varepsilon_{k}\right)_{k}$ is i.i.d. thus follows

$$
\mathbb{E}\left(\sum_{m=1}^{n-1}\left\|\boldsymbol{\delta}_{-m}^{(\mathfrak{p}, \mathfrak{q})}\right\|_{F^{\mathfrak{s}}}^{\nu} \prod_{l=1}^{m}\left\|\boldsymbol{\Psi}_{-m+l}^{(\mathfrak{p}, \mathfrak{q}}\right\|_{\mathcal{L}_{F^{\mathfrak{s}}}}^{\nu}\right)=\mathbb{E}\left\|\boldsymbol{\delta}_{0}^{(\mathfrak{p}, \mathfrak{q})}\right\|_{F^{\mathfrak{s}}}^{\nu} \sum_{m=1}^{n-1}\left(\mathbb{E}\left\|\Psi_{0}^{(\mathfrak{p}, \mathfrak{q})}\right\|_{\mathcal{L}_{F^{\mathfrak{s}}}}^{\nu}\right)^{m}<\infty
$$

Furthermore, (2.10) implies for $\nu \in(0,1]$ :

$$
\begin{aligned}
\mathbb{E}\left(\sum_{m=n}^{\infty}\left\|\Psi_{0}^{(\mathfrak{p}, \mathfrak{q})} \Psi_{-1}^{(\mathfrak{p}, \mathfrak{q})} \ldots \Psi_{-m+1}^{(\mathfrak{p}, \mathfrak{q})}\right\|_{\mathcal{L}_{F^{\mathfrak{s}}}}\left\|\boldsymbol{\delta}_{-m}^{(\mathfrak{p}, \mathfrak{q})}\right\|_{F^{\mathfrak{s}}}\right)^{\nu} \leq \mathbb{E}\left\|\boldsymbol{\delta}_{0}^{(\mathfrak{p}, \mathfrak{q})}\right\|_{F^{\mathfrak{s}}}^{\nu} \sum_{m=n}^{\infty} \mathbb{E}\left\|\boldsymbol{\Psi}_{0}^{(\mathfrak{p}, \mathfrak{q})} \mathbf{\Psi}_{-1}^{(\mathfrak{p}, \mathfrak{q})} \ldots \boldsymbol{\Psi}_{-m+1}^{(\mathfrak{p}, \mathfrak{q})}\right\|_{\mathcal{L}_{F^{\mathfrak{s}}}}^{\nu} \\
\leq \mathbb{E}\left\|\boldsymbol{\delta}_{0}^{(\mathfrak{p}, \mathfrak{q})}\right\|_{F^{\mathfrak{s}}}^{\nu}\left(\sum _ { k = 0 } ^ { n - 1 } \left(\mathbb{E}\left\|\boldsymbol{\Psi}_{0}^{(\mathfrak{p}, \mathfrak{q})}\right\|_{\left.\left.\mathcal{L}_{F^{\mathfrak{s}}}\right)^{k}\right) \sum_{l=1}^{\infty}\left(\psi_{n, \nu}^{(\mathfrak{p}, \mathfrak{q})}\right)^{l}<\infty}\right.\right.
\end{aligned}
$$

and for $\nu>1$ together with Jensen's inequality as well as monotone convergence theorem:

$$
\begin{aligned}
& \mathbb{E}\left(\sum_{m=n}^{\infty}\left\|\Psi_{0}^{(\mathfrak{p}, \mathfrak{q})} \Psi_{-1}^{(\mathfrak{p}, \mathfrak{q})} \cdots \boldsymbol{\Psi}_{-m+1}^{(\mathfrak{p}, \mathfrak{q})}\right\|_{\mathcal{L}_{F^{\mathfrak{s}}}}\left\|\boldsymbol{\delta}_{-m}^{(\mathfrak{p}, \mathfrak{q})}\right\|_{F^{\mathfrak{s}}}\right)^{\nu} \leq \mathbb{E}\left\|\boldsymbol{\delta}_{0}^{(\mathfrak{p}, \mathfrak{q})}\right\|_{F^{\mathfrak{s}}}^{\nu}\left(\sum_{m=n}^{\infty}\left(\mathbb{E}\left\|\mathbf{\Psi}_{0}^{(\mathfrak{p}, \mathfrak{q})} \boldsymbol{\Psi}_{-1}^{(\mathfrak{p}, \mathfrak{q})} \ldots \boldsymbol{\Psi}_{-m+1}^{(\mathfrak{p}, \mathfrak{q})}\right\|_{\mathcal{L}_{F^{\mathfrak{s}}}}^{\nu}\right)^{1 / \nu}\right)^{\nu} \\
& \leq \mathbb{E}\left\|\boldsymbol{\delta}_{0}^{(\mathfrak{p}, \mathfrak{q})}\right\|_{F^{\mathfrak{s}}}^{\nu}\left(\sum_{k=0}^{n-1}\left(\mathbb{E}\left\|\Psi_{0}^{(\mathfrak{p}, \mathfrak{q})}\right\|_{\mathcal{L}_{F^{\mathfrak{s}}}}^{\nu}\right)^{k}\right)\left(\sum_{l=1}^{\infty}\left(\psi_{n, \nu}^{(\mathfrak{p}, \mathfrak{q})}\right)^{l}\right)^{\nu}<\infty
\end{aligned}
$$


Subsequently, $\mathbb{E}\left\|\boldsymbol{\varsigma}_{0}^{(\mathfrak{p}, \mathfrak{q})}\right\|_{F^{\mathfrak{s}}}^{\nu}<\infty$ for all $\mathfrak{p} \in \mathbb{N}, \mathfrak{q} \in \mathbb{N}_{0}$ and thus $\mathbb{E}\left\|\mathscr{X}_{0}^{2}\right\|_{F}^{\nu}<\infty, \mathbb{E}\left\|\sigma_{0}^{2}\right\|_{F}^{\nu}<\infty$ as well as

$$
\begin{aligned}
\mathbb{E}|| \sigma_{0}^{2} \|_{\dot{F}}^{\nu} & \precsim\|\delta\|_{\dot{F}}^{\nu}+\sum_{i=1}^{\mathfrak{p}} \mathbb{E}|| \alpha_{i}\left(\mathscr{X}_{i}^{2}\right)\left\|_{\dot{F}}^{\nu}+\sum_{j=1}^{\mathfrak{q}} \mathbb{E}\right\| \beta_{j}\left(\sigma_{j}^{2}\right) \|_{\dot{F}}^{\nu} \\
& \leq\|\delta\|_{\dot{F}}^{\nu}+\mathbb{E}\left\|\mathscr{X}_{0}^{2}\right\|_{F}^{\nu} \sum_{i=1}^{\mathfrak{p}}\left\|\alpha_{i}\right\|_{\mathcal{L}_{F, \dot{F}}}^{\nu}+\mathbb{E}\left\|\sigma_{0}^{2}\right\|_{F}^{\nu} \sum_{j=1}^{\mathfrak{q}}\left\|\beta_{j}\right\|_{\mathcal{L}_{F, \dot{F}}}^{\nu}<\infty .
\end{aligned}
$$

(b) From the identity (5.2) follows

$$
\boldsymbol{\varsigma}_{m, m}^{(\mathfrak{p}, \mathfrak{q})}:=\boldsymbol{\delta}_{m}^{(\mathfrak{p}, \mathfrak{q})}+\sum_{l=1}^{m-1} \boldsymbol{\Psi}_{m}^{(\mathfrak{p}, \mathfrak{q})} \cdots \mathbf{\Psi}_{m-l+1}^{(\mathfrak{p}, \mathfrak{q})}\left(\boldsymbol{\delta}_{m-l}^{(\mathfrak{p}, \mathfrak{q})}\right)+\sum_{l=m}^{\infty} \boldsymbol{\Psi}_{m}^{(\mathfrak{p}, \mathfrak{q})} \cdots \mathbf{\Psi}_{1}^{(\mathfrak{p}, \mathfrak{q})} \mathbf{\Psi}_{0}^{(\mathfrak{p}, \mathfrak{q}, m)} \cdots \mathbf{\Psi}_{m-l+1}^{(\mathfrak{p}, \mathfrak{q}, m)}\left(\boldsymbol{\delta}_{m-l}^{(\mathfrak{p}, \mathfrak{q}, m)}\right)
$$

a.s. for all $m \in \mathbb{N}$. Thereby, $\Psi_{k}^{(\mathfrak{p}, \mathfrak{q}, m)}$ and $\boldsymbol{\delta}_{k}^{(\mathfrak{p}, \mathfrak{q}, m)}$ stand for $\boldsymbol{\Psi}_{k}^{(\mathfrak{p}, \mathfrak{q})}$ resp. $\boldsymbol{\delta}_{k}^{(\mathfrak{p}, \mathfrak{q})}$ in $(2.2)$ depending on $\varepsilon_{k}^{(m)}$ where $\left(\varepsilon_{k}^{(m)}\right)_{k \in \mathbb{Z}}$ are i.i.d. time series for all $m$, which are independent of each other, and $\varepsilon_{k}^{(m)}$ equals $\varepsilon_{0}$ in distribution for all $k, m$. Consequently, for any $m$,

$$
\begin{aligned}
\left\|\boldsymbol{\varsigma}_{m}^{(\mathfrak{p}, \mathfrak{q})}-\boldsymbol{\varsigma}_{m, m}^{(\mathfrak{p}, \mathfrak{q})}\right\|_{F^{\mathfrak{s}}} \leq \sum_{l=m}^{\infty}( & \left\|\mathbf{\Psi}_{m}^{(\mathfrak{p}, \mathfrak{q})} \ldots \mathbf{\Psi}_{m-l+1}^{(\mathfrak{p}, \mathfrak{q})}\right\|_{\mathcal{L}_{F^{\mathfrak{s}}}}\left\|\boldsymbol{\delta}_{m-l}^{(\mathfrak{p}, \mathfrak{q})}\right\|_{F^{\mathfrak{s}}} \\
& \left.+\left.\left\|\boldsymbol{\Psi}_{m}^{(\mathfrak{p}, \mathfrak{q})} \ldots \mathbf{\Psi}_{1}^{(\mathfrak{p}, \mathfrak{q})} \mathbf{\Psi}_{0}^{(\mathfrak{p}, \mathfrak{q}, m)} \ldots \mathbf{\Psi}_{m-l+1}^{(\mathfrak{p}, \mathfrak{q}, m)}\right\|\right|_{\mathcal{L}_{F^{\mathfrak{s}}}}\left\|\boldsymbol{\delta}_{m-l}^{(\mathfrak{p}, \mathfrak{q}, m)}\right\|_{F^{\mathfrak{s}}}\right) .
\end{aligned}
$$

From this identity, the proof of (a) and since $\varepsilon_{k}^{(n)}$ and $\varepsilon_{l}$ are i.i.d. for all $k, l, n$, it follows in the case $\nu \in(0,1]$ :

$$
\begin{aligned}
\mathbb{E}\left\|\boldsymbol{\varsigma}_{m}^{(\mathfrak{p}, \mathfrak{q})}-\boldsymbol{\varsigma}_{m, m}^{(\mathfrak{p}, \mathfrak{q})}\right\|_{F^{\mathfrak{s}}}^{\nu} & \leq 2 \mathbb{E}\left\|\boldsymbol{\delta}_{0}^{(\mathfrak{p}, \mathfrak{q})}\right\|_{F^{\mathfrak{s}}}^{\nu} \sum_{l=m}^{\infty} \mathbb{E}\left\|\mathbf{\Psi}_{m}^{(\mathfrak{p}, \mathfrak{q})} \cdots \mathbf{\Psi}_{m-l+1}^{(\mathfrak{p}, \mathfrak{q})}\right\|_{\mathcal{L}_{F^{\mathfrak{s}}}}^{\nu} \\
& \leq 2 \mathbb{E}\left\|\boldsymbol{\delta}_{0}^{(\mathfrak{p}, \mathfrak{q})}\right\|_{F^{\mathfrak{s}}}^{\nu}\left(\sum_{k=0}^{n-1}\left(\mathbb{E}\left\|\mathbf{\Psi}_{0}^{(\mathfrak{p}, \mathfrak{q})}\right\|_{\mathcal{L}_{F^{\mathfrak{s}}}}^{\nu}\right)^{k}\right) \sum_{j=m}^{\infty}\left(\psi_{n, \nu}^{(\mathfrak{p}, \mathfrak{q})}\right)^{j} \propto\left(\psi_{n, \nu}^{(\mathfrak{p}, \mathfrak{q})}\right)^{m}
\end{aligned}
$$

and in the case $\nu>1$, based on the argumentation in the proof of (a) for $\nu>1$ :

$$
\begin{aligned}
\mathbb{E}\left\|\boldsymbol{\varsigma}_{m}^{(\mathfrak{p}, \mathfrak{q})}-\boldsymbol{\varsigma}_{m, m}^{(\mathfrak{p}, \mathfrak{q})}\right\|_{F^{\mathfrak{s}}}^{\nu} & \leq\left(\sum_{m=n}^{\infty} 2\left(\mathbb{E}\left\|\mathbf{\Psi}_{m}^{(\mathfrak{p}, \mathfrak{q})} \cdots \mathbf{\Psi}_{m-l+1}^{(\mathfrak{p}, \mathfrak{q})}\right\|_{\mathcal{L}_{F^{\mathfrak{s}}}}^{\nu}\right)^{1 / \nu}\right)^{\nu} \\
& \leq 2^{\nu} \mathbb{E}\left\|\boldsymbol{\delta}_{0}^{(\mathfrak{p}, \mathfrak{q})}\right\|_{F^{\mathfrak{s}}}^{\nu}\left(\sum_{k=0}^{n-1}\left(\mathbb{E}\left\|\mathbf{\Psi}_{0}^{(\mathfrak{p}, \mathfrak{q})}\right\|_{\mathcal{L}_{F^{\mathfrak{s}}}}^{\nu}\right)^{k}\right)\left(\sum_{j=m}^{\infty}\left(\psi_{n, \nu}^{(\mathfrak{p}, \mathfrak{q})}\right)^{j / \nu}\right)^{\nu} \propto\left(\psi_{n, \nu}^{(\mathfrak{p}, \mathfrak{q})}\right)^{m / \nu}
\end{aligned}
$$

Proof of Lemma 3.1. See Kühnert (2019), Lemma 4.11.

Proof of Lemma 3.2. See Kühnert (2019), Theorem 4.2.

Proof of Theorem 3.1. See Kühnert (2019), Theorem 4.4.

Proof of Theorem 3.2. The assertion follows from (3.6) and

$$
\|\hat{\delta}-\delta\|_{\mathscr{H}} \leq\left\|\hat{m}_{2}-m_{2}\right\|_{\mathscr{H}}\left(1+\sum_{i=1}^{\mathfrak{r}}\left\|\alpha_{i}+\beta_{i}\right\|_{\mathcal{L}_{\mathscr{H}}}\right)+\left\|\hat{m}_{2}\right\| \|_{\mathscr{H}}\left(\sum_{i=1}^{\mathfrak{r}}\left\|\hat{\alpha}_{i}-\alpha_{i}\right\|_{\mathcal{L}_{\mathscr{H}}}+\left\|\hat{\beta}_{i}-\beta_{i}\right\|_{\mathcal{L}_{\mathscr{H}}}\right) .
$$

Proof of Theorem 3.3. (a) The definition of $\hat{\boldsymbol{\alpha}}_{[\mathfrak{p}]}$, the inequality $(5.1), \mathfrak{S}_{\mathfrak{p}}^{\dagger}:=\mathfrak{S}_{\mathfrak{p}}\left(\mathfrak{S}_{\mathfrak{p}}^{2}+\vartheta_{N} \mathbb{I}_{\mathscr{H}}\right)^{-1}$, the YuleWalker equation $\mathfrak{S}_{\mathfrak{p}, 1}=\boldsymbol{\alpha} \mathfrak{S}_{\mathfrak{p}}, \mathfrak{S}_{\mathfrak{p}}^{\ddagger}:=\mathfrak{S}_{\mathfrak{p}} \mathfrak{S}_{\mathfrak{p}}^{\dagger},(3.10), \mathbb{I}_{\mathscr{H}} \mathfrak{p}=\coprod_{\hat{\mathfrak{c}}_{\mathrm{p}, 1}}^{\hat{\mathfrak{p}}_{\mathrm{p}}}+\coprod_{\hat{\mathfrak{p}}_{\mathrm{p}, K+1}}^{\hat{\mathfrak{c}}_{\mathfrak{p}, \infty}}$ and $\boldsymbol{\alpha}=\coprod_{\mathscr{F}_{\mathrm{p}, K}} \boldsymbol{\alpha}+\coprod_{\mathscr{f}_{\mathrm{p}, K}^{c}} \boldsymbol{\alpha}$ with $\mathscr{J}_{\mathfrak{p}, K}:=\left\{\Phi_{\mathfrak{p}, i j} \mid 1 \leq i, j \leq K\right\}$ and $\mathscr{J}_{\mathfrak{p}, K}^{c}:=\left\{\Phi_{\mathfrak{p}, i j} \mid i, j \in \mathbb{N}, i \vee j>K\right\}$, yields for fixed $K \in \mathbb{N}$ :

$$
\left\|\hat{\boldsymbol{\alpha}}_{[\mathfrak{p}]}-\coprod_{\mathscr{J}_{\mathfrak{p}, K}} \boldsymbol{\alpha}_{[\mathfrak{p}]}\right\|_{\mathcal{S}_{\mathscr{H} \mathfrak{p}, \mathscr{H}}}^{2} \precsim\left\|\left(\hat{\mathfrak{S}}_{\mathfrak{p}, 1}-\mathfrak{S}_{\mathfrak{p}, 1}\right) \hat{\mathfrak{S}}_{\mathfrak{p}}^{\dagger} \coprod_{\hat{\mathfrak{c}}_{\mathfrak{p}, 1}}^{\hat{\mathfrak{p}}_{\mathfrak{p}, K}}\right\|_{\mathcal{S}_{\mathscr{H}}, \mathfrak{H},}^{2}+\left\|\mathfrak{S}_{\mathfrak{p}, 1} \hat{\mathfrak{S}}_{\mathfrak{p}}^{\dagger} \coprod_{\mathfrak{c}_{\mathfrak{p}, 1}}^{\mathfrak{c}_{\mathfrak{p}, K}}-\coprod_{\mathscr{J}_{\mathfrak{p}, K}} \boldsymbol{\alpha}_{[\mathfrak{p}]}\right\|_{\mathcal{S}_{\mathscr{H}} \mathfrak{p}, \mathscr{H}}^{2}
$$




$$
\begin{aligned}
& \precsim\left\|\hat{\mathfrak{S}}_{\mathfrak{p}, 1}-\mathfrak{S}_{\mathfrak{p}, 1}\right\|_{\mathcal{S}_{\mathscr{H}} \mathfrak{p}, \mathscr{H}}^{2}\left\|\hat{\mathfrak{S}}_{\mathfrak{p}}^{\dagger} \coprod_{\hat{\mathfrak{c}}_{\mathfrak{p}, 1}}^{\hat{\mathfrak{c}}_{\mathfrak{p}, K}}\right\|_{\mathcal{L}_{\mathscr{H}} \mathfrak{p}}^{2}+\left\|\mathfrak{S}_{\mathfrak{p}, 1}\right\|_{\mathcal{S}_{\mathscr{H}} \mathfrak{p}, \mathscr{H}}^{2}\left\|\hat{\mathfrak{S}}_{\mathfrak{p}}^{\dagger} \coprod_{\hat{\mathfrak{c}}_{\mathfrak{p}, 1}}^{\hat{\mathfrak{c}}_{\mathfrak{p}, K}}-\mathfrak{S}_{\mathfrak{p}}^{\dagger} \coprod_{\mathfrak{c}_{\mathfrak{p}, 1}}^{\mathfrak{c}_{\mathfrak{p}, K}}\right\|_{\mathcal{L}_{\mathscr{H}} \mathfrak{p}}^{2} \\
& +\left\|\boldsymbol{\alpha}_{[\mathfrak{p}]} \mathfrak{S}_{\mathfrak{p}}^{\ddagger} \coprod_{\mathfrak{c}_{\mathfrak{p}, 1}}^{\mathfrak{c}_{\mathfrak{p}, K}}-\coprod_{\mathscr{J}_{\mathfrak{p}, K}} \boldsymbol{\alpha}_{[\mathfrak{p}]}\right\|_{\mathcal{S}_{\mathscr{H}}, \mathscr{H}}^{2} \\
& \precsim \mathrm{O}_{\mathbb{P}}\left(N^{-1}\right)\left\|\hat{\mathfrak{S}}_{\mathfrak{p}}^{\dagger} \coprod_{\hat{\mathfrak{c}}_{\mathfrak{p}, 1}}^{\hat{\mathfrak{c}}_{\mathfrak{p}, K}}\right\|_{\mathcal{L}_{\mathscr{H}} \mathfrak{p}}^{2}+\left\|\hat{\mathfrak{S}}_{\mathfrak{p}}^{\dagger} \coprod_{\hat{\mathfrak{c}}_{\mathfrak{p}, 1}}^{\hat{\mathfrak{c}}_{\mathfrak{p}, K}}-\mathfrak{S}_{\mathfrak{p}}^{\dagger} \coprod_{\mathfrak{c}_{\mathfrak{p}, 1}}^{\mathfrak{c}_{\mathfrak{p}, K}}\right\|_{\mathcal{L}_{\mathscr{H}} \mathfrak{p}}^{2}+\left\|\coprod_{\mathscr{J}_{\mathfrak{p}, K}^{c}} \boldsymbol{\alpha}_{[\mathfrak{p}]} \mathfrak{S}_{\mathfrak{p}}^{\ddagger} \coprod_{\mathfrak{c}_{\mathfrak{p}, 1}}^{\mathfrak{c}_{\mathfrak{p}, K}}\right\|_{\mathcal{S}_{\mathscr{H}} \mathfrak{p}, \mathscr{H}}^{2} \\
& +\left\|\coprod_{\mathscr{J}_{\mathfrak{p}, K}} \boldsymbol{\alpha}_{[\mathfrak{p}]}\left[\mathfrak{S}_{\mathfrak{p}}^{\ddagger} \coprod_{\mathfrak{c}_{\mathfrak{p}, 1}}^{\mathfrak{c}_{\mathfrak{p}, K}}-\mathbb{I}_{\mathscr{H}^{\mathfrak{p}}}\right]\right\|_{\mathcal{S}_{\mathscr{H}} \mathfrak{p}, \mathscr{H}}^{2} \\
& =: \mathrm{O}_{\mathbb{P}}\left(N^{-1}\right) \cdot T_{1}+T_{2}+T_{3}+T_{4} \text {. }
\end{aligned}
$$

$\underline{\text { Term } T_{1}}$ : As per definition of $\|\cdot\|_{\mathcal{L}_{\mathscr{H}}}, \hat{\mathfrak{S}}_{\mathfrak{p}}^{\dagger}:=\hat{\mathfrak{S}}_{\mathfrak{p}}\left(\hat{\mathfrak{S}}_{\mathfrak{p}}^{2}+\vartheta_{N} \mathbb{I}_{\mathscr{H}}\right)^{-1}$, since $\left(\hat{\mathfrak{c}}_{\mathfrak{p}, j}\right)_{j}$ is the eigenfunction sequence

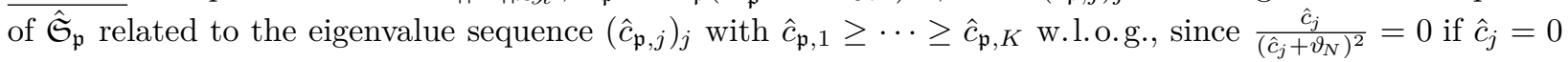
resp. $\frac{\hat{c}_{j}}{\left(\hat{c}_{j}+\vartheta_{N}\right)^{2}} \leq \hat{c}_{j}^{-1}$ if $\hat{c}_{j} \neq 0$ and because of (3.15) with $K_{N}:=K$ for all $N$, we have

$$
\left\|\hat{\mathfrak{S}}_{\mathfrak{p}}^{\dagger} \coprod_{\hat{\mathfrak{c}}_{\mathfrak{p}, 1}}^{\hat{\mathfrak{c}}_{\mathfrak{p}, K}}\right\|_{\mathcal{L}_{\mathscr{H}} \mathfrak{p}}^{2}=\sup _{j \leq K}\left(\frac{\hat{c}_{\mathfrak{p}, j}}{\hat{c}_{\mathfrak{p}, j}+\vartheta_{N}}\right)^{2}=\mathrm{O}_{\mathbb{P}}\left(c_{\mathfrak{p}, K}^{-2}\right)=\mathrm{O}_{\mathbb{P}}(1)
$$

$\underline{\text { Term } T_{2}}$ : From the definition of $\hat{\mathfrak{S}}_{\mathfrak{p}}^{\dagger}, \mathfrak{S}_{\mathfrak{p}}^{\dagger}$ and $\|\cdot\|_{\mathcal{L}_{\mathscr{e}} \mathfrak{p}}$, since $\left(\mathfrak{c}_{\mathfrak{p}, j}\right)_{j}$ is a CONS of $\mathscr{H}^{\mathfrak{p}}$ and $\left(\hat{\mathfrak{c}}_{\mathfrak{p}, j}^{\prime \prime \prime}\right)_{j}$ is a CONS of $\mathscr{H}^{\mathfrak{p}}$ a.s., Corollary 3.1 and Lemma 3.3 imply

$$
\begin{aligned}
\left\|\hat{\mathfrak{S}}_{\mathfrak{p}}^{\dagger} \coprod_{\hat{\mathfrak{c}}_{\mathfrak{p}, 1}}^{\hat{\mathfrak{c}}_{\mathfrak{p}, K}}-\mathfrak{S}_{\mathfrak{p}}^{\dagger} \coprod_{\mathfrak{c}_{\mathfrak{p}, 1}}^{\mathfrak{c}_{\mathfrak{p}, K}}\right\|_{\mathcal{L}_{\mathscr{H}^{\mathfrak{p}}}}^{2} & =\sup _{\|\boldsymbol{x}\| \|_{\mathscr{H}} \leq 1}\left\|\sum_{j=1}^{K} \frac{\hat{\mathfrak{c}}_{\mathfrak{p}, j}}{\hat{c}_{\mathfrak{p}, j}^{2}+\vartheta_{N}}\left\langle\boldsymbol{x}, \hat{\mathfrak{c}}_{\mathfrak{p}, j}^{\prime \prime \prime}\right\rangle_{\mathscr{H}^{\mathfrak{p}}} \hat{\mathfrak{c}}_{\mathfrak{p}, j}^{\prime \prime \prime}-\frac{c_{\mathfrak{p}, j}}{c_{\mathfrak{p}, j}^{2}+\vartheta_{N}}\left\langle\boldsymbol{x}, \mathfrak{c}_{\mathfrak{p}, j}\right\rangle_{\mathscr{H}^{\mathfrak{p}}} \mathfrak{c}_{\mathfrak{p}, j}\right\|_{\mathscr{H}^{\mathfrak{p}}}^{2} \\
& \precsim \sup _{j \leq K}\left|\frac{\hat{c}_{\mathfrak{p}, j}}{\hat{c}_{\mathfrak{p}, j}^{2}+\vartheta_{N}}-\frac{c_{\mathfrak{p}, j}}{c_{\mathfrak{p}, j}^{2}+\vartheta_{N}}\right|^{2}+\sup _{\|\boldsymbol{x}\|_{\mathscr{H}^{\mathfrak{p}} \leq 1}} \sum_{j=1}^{K}\left|\frac{c_{\mathfrak{p}, j}}{c_{\mathfrak{p}, j}^{2}+\vartheta_{N}}\right|^{2}\left\langle\boldsymbol{x}, \hat{\mathfrak{c}}_{\mathfrak{p}, j}^{\prime \prime \prime}-\mathfrak{c}_{\mathfrak{p}, j}\right\rangle_{\mathscr{H}^{\mathfrak{p}}}^{2} \\
& +\sup _{\|\boldsymbol{x}\|_{\mathscr{H}} \leq 1} \|\left.\sum_{j=1}^{K} \frac{c_{\mathfrak{p}, j}}{c_{\mathfrak{p}, j}^{2}+\vartheta_{N}}\left\langle\boldsymbol{x}, \mathfrak{c}_{\mathfrak{p}, j}\right\rangle_{\mathscr{H}_{\mathfrak{p}}}\left(\hat{\mathfrak{c}}_{\mathfrak{p}, j}^{\prime \prime \prime}-\mathfrak{c}_{\mathfrak{p}, j}\right)\right|_{\mathscr{H}^{\mathfrak{p}}} ^{2} \\
& \precsim \sup _{j \leq K}\left(\hat{c}_{\mathfrak{p}, j}-c_{\mathfrak{p}, j}\right)^{2} \frac{\vartheta_{N}^{2}+\hat{c}_{\mathfrak{p}, j}^{2} c_{\mathfrak{p}, j}^{2}}{\left(\hat{c}_{\mathfrak{p}, j}^{2}+\vartheta_{N}\right)^{2}\left(c_{\mathfrak{p}, j}^{2}+\vartheta_{N}\right)^{2}}+(K+1) c_{\mathfrak{p}, K}^{-2} \sup _{j \leq K}\left\|\hat{\mathfrak{c}}_{\mathfrak{p}, j}^{\prime \prime \prime}-\mathfrak{c}_{\mathfrak{p}, j}\right\|_{\mathscr{H}^{\mathfrak{p}}}^{2} \\
= & \mathrm{O}_{\mathbb{P}}\left(c_{\mathfrak{p}, K}^{-4} N^{-1}\right)+\mathrm{O}_{\mathbb{P}}\left(c_{\mathfrak{p}, K}^{-4} K N^{-1}\right)=\mathrm{O}_{\mathbb{P}}\left(N^{-1}\right) .
\end{aligned}
$$

Term $T_{3}$ : The eigenfunction sequences $\left(\mathfrak{c}_{\mathfrak{p}, j}\right)_{j}$ of $\mathfrak{S}_{\mathfrak{p}},\left(\mathfrak{c}_{j}\right)_{j}$ of $\mathscr{C}_{0 ; \mathscr{Z}}$ and the sequence $\left(\Phi_{\mathfrak{p}, i j}\right)_{i, j}$ with $\Phi_{\mathfrak{p}, i j}\left(\mathfrak{c}_{\mathfrak{p}, k}\right)=$


$\left\{\Phi_{\mathfrak{p}, i j} \mid i, j \in \mathbb{N}, i \vee j>K\right\}, \boldsymbol{\alpha}_{[\mathfrak{p}]}=\sum_{i, j=1}^{\infty}\left\langle\boldsymbol{\alpha}_{[\mathfrak{p}]}, \Phi_{\mathfrak{p}, i j}\right\rangle_{\mathcal{S}_{\mathscr{C}, \mathfrak{p}, ~}} \Phi_{\mathfrak{p}, i j},\left\langle\boldsymbol{\alpha}_{[\mathfrak{p}]}, \Phi_{\mathfrak{p}, i j}\right\rangle_{\mathcal{S}_{\mathscr{H}, ~}, \mathscr{C}}=\sum_{k=1}^{\infty}\left\langle\boldsymbol{\alpha}_{[\mathfrak{p}]}\left(\mathfrak{c}_{\mathfrak{p}, k}\right), \Phi_{\mathfrak{p}, i j}\left(\mathfrak{c}_{\mathfrak{p}, k}\right)\right\rangle_{\mathscr{H}}=$ $\left\langle\boldsymbol{\alpha}_{[\mathfrak{p}]}\left(\mathfrak{c}_{\mathfrak{p}, i}\right), \mathfrak{c}_{j}\right\rangle_{\mathscr{H}}$ for all $i, j$ and since we imposed $\left\langle\boldsymbol{\alpha}_{[\mathfrak{p}]}\left(\mathfrak{c}_{\mathfrak{p}, l}\right), \mathfrak{c}_{j}\right\rangle_{\mathscr{H}}=0$ for all $j>K, l \leq K$, we thus obtain

$$
\begin{aligned}
\left\|\coprod_{\mathscr{J}_{\mathfrak{p}, K}^{c}} \boldsymbol{\alpha}_{[\mathfrak{p}]} \mathfrak{S}_{\mathfrak{p}}^{\ddagger} \coprod_{\mathfrak{c}_{\mathfrak{p}, 1}^{\ddagger}}^{\mathfrak{c}_{\mathfrak{p}, K}}\right\|_{\mathcal{S}_{\mathscr{H}, \mathfrak{H}, ~}}^{2} & =\sum_{l=1}^{K}\left\|\coprod_{\mathcal{J}_{\mathfrak{p}, K}^{c}} \boldsymbol{\alpha}_{[\mathfrak{p}]} \mathfrak{S}_{\mathfrak{p}}^{\ddagger}\left(\mathfrak{c}_{\mathfrak{p}, l}\right)\right\|_{\mathscr{H}}^{2}=\sum_{l=1}^{K}\left(\frac{c_{\mathfrak{p}, l}^{2}}{c_{\mathfrak{p}, l}^{2}+\vartheta_{N}}\right)^{2}\left\|\coprod_{\mathscr{J}_{\mathfrak{p}, K}^{c}} \boldsymbol{\alpha}_{[\mathfrak{p}]}\left(\mathfrak{c}_{\mathfrak{p}, l}\right)\right\|_{\mathscr{H}}^{2} \\
& =\sum_{l=1}^{K}\left(\frac{c_{\mathfrak{p}, l}^{2}}{c_{\mathfrak{p}, l}^{2}+\vartheta_{N}}\right)^{2}\left\|\sum_{\substack{i, j \in \mathbb{N} \\
i \vee j>K}}\left\langle\boldsymbol{\alpha}_{[\mathfrak{p}]}\left(\mathfrak{c}_{\mathfrak{p}, i}\right), \mathfrak{c}_{j}\right\rangle_{\mathscr{H}} \delta_{i l} \mathfrak{c}_{j}\right\|_{\mathscr{H}}^{2}=\sum_{l=1}^{K}\left(\frac{c_{\mathfrak{p}, l}^{2}}{c_{\mathfrak{p}, l}^{2}+\vartheta_{N}}\right)^{2} \sum_{j>K}\left\langle\boldsymbol{\alpha}_{[\mathfrak{p}]}\left(\mathfrak{c}_{\mathfrak{p}, l}\right), \mathfrak{c}_{j}\right\rangle_{\mathscr{H}}^{2} \\
& =0 .
\end{aligned}
$$

$\underline{\text { Term } T_{4}}$ : Elementary transformations and transformations as used in the terms $T_{1}-T_{3}, \coprod_{\mathscr{J}_{\mathfrak{p}, K}} \boldsymbol{\alpha}_{[\mathfrak{p}]}\left(\mathfrak{c}_{\mathfrak{p}, l}\right)=$ 
$\mathbf{1}_{\mathbb{N}_{\leq K}}(l) \sum_{j=1}^{K}\left\langle\boldsymbol{\alpha}_{[\mathfrak{p}]}\left(\mathfrak{c}_{\mathfrak{p}, l}\right), \mathfrak{c}_{j}\right\rangle_{\mathscr{H}} \mathfrak{c}_{j}$ for all $l, K$ and $\vartheta_{N}=\mathrm{O}\left(N^{-1 / 2}\right)$ imply

$$
\begin{aligned}
\left\|\coprod_{\mathscr{J}_{\mathfrak{p}, K}} \boldsymbol{\alpha}_{[\mathfrak{p}]}\left[\mathfrak{S}_{\mathfrak{p}}^{\ddagger} \coprod_{\mathfrak{c}_{\mathfrak{p}, 1}}^{\mathfrak{c}_{\mathfrak{p}, K}}-\mathbb{I}_{\mathscr{H} \mathfrak{p}}\right]\right\|_{\mathcal{S}_{\mathscr{H}, \mathfrak{p},}}^{2} & =\sum_{l=1}^{\infty}\left\|\coprod_{\mathscr{J}_{\mathfrak{p}, K}} \boldsymbol{\alpha}_{[\mathfrak{p}]}\left(\left[\mathbf{1}_{\mathbb{N}_{\leq K}}(l) \frac{c_{\mathfrak{p}, l}^{2}}{c_{\mathfrak{p}, l}^{2}+\vartheta_{N}}-1\right] \mathfrak{c}_{\mathfrak{p}, l}\right)\right\|_{\mathscr{H}}^{2} \\
& =\sum_{l=1}^{\infty}\left\|\left[\mathbf{1}_{\mathbb{N}_{\leq K}}(l) \frac{c_{\mathfrak{p}, l}^{2}}{c_{\mathfrak{p}, l}^{2}+\vartheta_{N}}-1\right] \mathbf{1}_{\mathbb{N}_{\leq K}}(l) \sum_{j=1}^{K}\left\langle\boldsymbol{\alpha}_{[\mathfrak{p}]}\left(\mathfrak{c}_{\mathfrak{p}, l}\right), \mathfrak{c}_{j}\right\rangle_{\mathscr{H}} \mathfrak{c}_{j}\right\|_{\mathscr{H}}^{2} \\
& =\sum_{j, l=1}^{K}\left(\frac{\vartheta_{N}}{c_{\mathfrak{p}, l}^{2}+\vartheta_{N}}\right)^{2}\left\langle\boldsymbol{\alpha}_{[\mathfrak{p}]}\left(\mathfrak{c}_{\mathfrak{p}, l}\right), \mathfrak{c}_{j}\right\rangle_{\mathscr{H}}^{2} \leq \vartheta_{N}^{2} c_{\mathfrak{p}, K}^{-4}\left\|\boldsymbol{\alpha}_{[\mathfrak{p}]}\right\|_{\mathcal{S}_{\mathscr{H}}, \mathscr{H}}^{2} \\
& =\mathrm{O}\left(N^{-1}\right) .
\end{aligned}
$$

Replacing $T_{1}-T_{4}$ in (5.3) by (5.4)-(5.7) indeed yields (3.33).

(b) follows from $\vartheta_{N}=\mathrm{O}\left(N^{-1 / 2}\right), c_{\mathfrak{p}, K}^{-4} K^{2 \beta+1}=\mathrm{O}(N)$ and $\sum_{l=1}^{K}\left(\frac{c_{\mathfrak{p}, l}^{2}}{c_{\mathfrak{p}, l}^{2}+\vartheta_{N}}\right)^{2} \sum_{j>K}\left\langle\boldsymbol{\alpha}_{[\mathfrak{p}]}\left(\mathfrak{c}_{\mathfrak{p}, l}\right), \mathfrak{c}_{j}\right\rangle_{\mathscr{H}}^{2}=\mathrm{O}\left(K^{-2 \beta}\right)$ for any sequence $K=K_{N}=\Xi(1, N)$, and the fact that $\boldsymbol{\alpha}_{[\mathfrak{p}]}=\coprod_{\mathscr{J}_{\mathfrak{p}, K}} \boldsymbol{\alpha}_{[\mathfrak{p}]}+\coprod_{\mathscr{J}_{\mathfrak{p}, K}^{c}} \boldsymbol{\alpha}_{[\mathfrak{p}]}$, (a) and (3.26) imply

$$
\begin{aligned}
& \left\|\hat{\boldsymbol{\alpha}}_{[\mathfrak{p}]}-\boldsymbol{\alpha}_{[\mathfrak{p}]}\right\|_{\mathcal{S}_{\mathscr{H}} \mathfrak{p}, \mathscr{H}}^{2} \precsim\left\|\hat{\boldsymbol{\alpha}}_{[\mathfrak{p}]}-\coprod_{\mathscr{J}_{\mathfrak{p}, K}} \boldsymbol{\alpha}_{[\mathfrak{p}]}\right\|_{\mathcal{S}_{\mathscr{H}}, \mathscr{H}}^{2}+\left\|\coprod_{\mathscr{J}_{\mathfrak{p}, K}^{c}} \boldsymbol{\alpha}_{[\mathfrak{p}]}\right\|_{\mathcal{S}_{\mathscr{H}}, \mathscr{H}}^{2} \\
& \precsim \mathrm{O}_{\mathbb{P}}\left(c_{\mathfrak{p}, K}^{-2} N^{-1}\right)+\mathrm{O}_{\mathbb{P}}\left(c_{\mathfrak{p}, K}^{-4} N^{-1}\right)+\mathrm{O}_{\mathbb{P}}\left(c_{\mathfrak{p}, K}^{-4} K N^{-1}\right)+ \\
& +\sum_{l=1}^{K}\left(\frac{c_{\mathfrak{p}, l}^{2}}{c_{\mathfrak{p}, l}^{2}+\vartheta_{N}}\right)^{2} \sum_{j>K}\left\langle\boldsymbol{\alpha}_{[\mathfrak{p}]}\left(\mathfrak{c}_{\mathfrak{p}, l}\right), \mathfrak{c}_{j}\right\rangle_{\mathscr{H}}^{2}+\vartheta_{N}^{2} c_{\mathfrak{p}, K}^{-4}\left\|\boldsymbol{\alpha}_{[\mathfrak{p}]}\right\|_{\mathcal{S}_{\mathscr{H}} \mathfrak{p}, \mathscr{H}}^{2}+\mathrm{O}\left(K^{-2 \beta}\right) \text {. }
\end{aligned}
$$

Proof of Theorem 3.4. The proof is based on the proof of Theorem 3.3 with $\mathfrak{p}$ replaced by an appropriate sequence $L=L_{N} \rightarrow \infty$. From the ideas in the proof of Theorem 3.3 and (see Kühnert (2019), Lemma 4.45)

$$
\mathfrak{S}_{L, 1}=\pi_{L} \mathfrak{S}_{L}+\sum_{l>L} \pi_{l} \mathfrak{S}_{L, 1-l}
$$

which can be identified as a Yule-Walker equation with a residual, follows for all $K, L, N$ :

$$
\begin{aligned}
& \left\|\hat{\pi}_{L, K}-\pi_{L}\right\|_{\mathcal{S}_{\mathscr{H} L}, \mathscr{H}}^{2} \precsim\left\|\hat{\pi}_{L, K}-\coprod_{\mathscr{J}_{L, K}} \pi_{L}\right\|_{\mathcal{S}_{\mathscr{H}^{L}, \mathscr{H}}}^{2}+\left\|\coprod_{\mathscr{J}_{L, K}^{c}} \pi_{L}\right\|_{\mathcal{S}_{\mathscr{H}^{L}, \mathscr{H}}}^{2} \\
& \precsim\left\|\hat{\mathfrak{S}}_{L, 1}-\mathfrak{S}_{L, 1}\right\|_{\mathcal{S}_{\mathscr{H}^{L}, \mathscr{H}}}^{2}\left\|\hat{\mathfrak{S}}_{L}^{\dagger} \coprod_{\hat{\mathfrak{c}}_{L, 1}}^{\hat{\mathfrak{c}}_{L, K}}\right\|_{\mathcal{L}_{\mathscr{H}_{L}}}^{2}+\left\|\mathfrak{S}_{L, 1}\right\|_{\mathcal{S}_{\mathscr{H} L}, \mathscr{H}}^{2}\left\|\hat{\mathfrak{S}}_{L}^{\dagger} \coprod_{\hat{\mathfrak{c}}_{L, 1}}^{\hat{\mathfrak{c}}_{L, K}}-\mathfrak{S}_{L}^{\dagger} \coprod_{\mathfrak{c}_{L, 1}}^{\mathfrak{c}_{L, K}}\right\|_{\mathcal{L}_{\mathscr{H}^{L}}}^{2} \\
& +\left\|\mathfrak{S}_{L, 1} \mathfrak{S}_{L}^{\dagger} \coprod_{\mathfrak{c}_{L, 1}}^{\mathfrak{c}_{L, K}}-\coprod_{\mathscr{J}_{L, K}} \boldsymbol{\pi}_{L}\right\|_{\mathcal{S}_{\mathscr{H}}, \mathscr{H}}^{2}+\mathrm{O}\left(K^{-2 \beta}\right) \\
& \precsim \mathrm{O}_{\mathbb{P}}\left(L^{2} N^{-1}\right)\left\|\hat{\mathfrak{S}}_{L}^{\dagger} \coprod_{\hat{\mathfrak{c}}_{L, 1}}^{\hat{\mathfrak{c}}_{L, K}}\right\|_{\mathcal{L}_{\mathscr{H} L}}^{2}+L \cdot\left\|\hat{\mathfrak{S}}_{L}^{\dagger} \coprod_{\hat{\mathfrak{c}}_{L, 1}}^{\hat{\mathfrak{c}}_{L, K}}-\mathfrak{S}_{L}^{\dagger} \coprod_{\mathfrak{c}_{L, 1}}^{\mathfrak{c}_{L, K}}\right\|_{\mathcal{L}_{\mathscr{H} L}}^{2}+\left\|\left[\sum_{l>L} \pi_{l} \mathfrak{S}_{L, 1-l}\right] \mathfrak{S}_{L}^{\dagger} \coprod_{\mathfrak{c}_{L, 1}}^{\mathfrak{c}_{L, K}}\right\|_{\mathcal{S}_{\mathscr{H} L}, \mathscr{H}}^{2} \\
& +\left\|\coprod_{\mathscr{J}_{L, K}^{c}} \pi_{L} \mathfrak{S}_{L}^{\ddagger} \coprod_{\mathfrak{c}_{L, 1}}^{\mathfrak{c}_{L, K}}\right\|_{\mathcal{S}_{\mathscr{H} L}, \mathscr{H}}^{2}+\left\|\coprod_{\mathscr{J}_{L, K}} \pi_{L}\left[\mathfrak{S}_{L}^{\ddagger} \coprod_{\mathfrak{c}_{L, 1}}^{\mathfrak{c}_{L, K}}-\mathbb{I}_{\mathscr{H}^{L}}\right]\right\|_{\mathcal{S}_{\mathscr{H} L}, \mathscr{H}}^{2}+\mathrm{O}\left(K^{-2 \beta}\right) \\
& =: \mathrm{O}_{\mathbb{P}}\left(L^{2} N^{-1}\right) \cdot T_{1}+L \cdot T_{2}+T_{3}+T_{4}+T_{5}+\mathrm{O}\left(K^{-2 \beta}\right) .
\end{aligned}
$$

$\underline{\text { Term } T_{1}}$ : Conversions similar as in $T_{1}$ in the proof of Theorem 3.3 and Corollary 3.1 yield

$$
\left\|\hat{\mathfrak{S}}_{L}^{\dagger} \coprod_{\hat{\mathfrak{c}}_{L, 1}}^{\hat{\mathfrak{c}}_{L, K}}\right\|_{\mathcal{L}_{\mathscr{H}} L}^{2}=\mathrm{O}_{\mathbb{P}}\left(c_{L, K}^{-2}\right)
$$


$\underline{\text { Term } T_{2}}$ : (3.12) with $L=\Xi\left(1, N^{1 / 3}\right)$ and the argumentation of $T_{2}$ in the proof of Theorem 3.3 yield

$$
\left\|\hat{\mathfrak{S}}_{L}^{\dagger} \coprod_{\hat{\mathfrak{c}}_{L, 1}}^{\hat{\mathfrak{c}}_{L, K}}-\mathfrak{S}_{L}^{\dagger} \coprod_{\mathfrak{c}_{L, 1}}^{\mathfrak{c}_{L, K}}\right\|_{\mathcal{L}_{\mathscr{H}} L}^{2}=\mathrm{O}_{\mathbb{P}}\left(c_{L, K}^{-4} L^{3} N^{-1}\right)+\mathrm{O}_{\mathbb{P}}\left(c_{L, K}^{-4} K L^{3} N^{-1}\right)=\mathrm{O}_{\mathbb{P}}\left(c_{L, K}^{-4} K L^{3} N^{-1}\right) .
$$

$\underline{\text { Term } T_{3}}$ : From the operator-valued Hölder's inequality (5.1), (5.10), triangle inequality and (3.8) follows

$$
\left\|\left[\sum_{l>L} \pi_{l} \mathfrak{S}_{L, 1-l}\right] \mathfrak{S}_{L}^{\dagger} \coprod_{\mathfrak{c}_{L, 1}}^{\mathfrak{c}_{L, K}}\right\|_{\mathcal{S}_{\mathscr{H}}, \mathscr{H}}^{2} \leq c_{L, K}^{-2}\left\|\sum_{l>L} \pi_{l} \mathfrak{S}_{L, 1-l}\right\|_{\mathcal{S}_{\mathscr{H}}, \mathscr{H}}^{2}=\mathrm{O}\left(L\left(c_{L, K}^{-1} \sum_{l>L}\left\|\pi_{l}\right\|_{\left.\mathcal{L}_{\mathscr{H}}\right)^{2}}\right)\right.
$$

Term $T_{4}$ : Here, almost one to one as in the proof of Theorem 3.3, we obtain by assumption

$$
\left\|\coprod_{\mathscr{J}_{L, K}^{c}} \boldsymbol{\pi}_{L} \mathfrak{S}_{L}^{\ddagger} \coprod_{\mathfrak{c}_{L, 1}}^{\mathfrak{c}_{L, K}}\right\|_{\mathcal{S}_{\mathscr{H}, ~}, \mathscr{H}}^{2}=\sum_{l=1}^{K}\left(\frac{c_{L, l}^{2}}{c_{L, l}^{2}+\vartheta_{N}}\right)^{2} \sum_{j>K}\left\langle\boldsymbol{\pi}_{L}\left(\mathfrak{c}_{L, l}\right), \mathfrak{c}_{j}\right\rangle_{\mathscr{H}}^{2}=\mathrm{O}\left(K^{-2 \beta}\right) .
$$

$\underline{\text { Term } T_{5}}:\left\|\boldsymbol{\pi}_{L}\right\|_{\mathcal{S}_{\mathscr{H}} L \mathscr{H}}^{2}=\sum_{l=1}^{L}\left\|\pi_{l}\right\|_{\mathcal{S}_{\mathscr{H}}}^{2} \leq \sum_{l=1}^{\infty}\left\|\pi_{l}\right\|_{\mathcal{S}_{\mathscr{H}}}^{2}<\infty$ implies as in $T_{4}$ in the proof of Theorem 3.3:

$$
\left\|\coprod_{\mathscr{J}_{L, K}} \boldsymbol{\pi}_{L}\left[\mathfrak{S}_{L}^{\ddagger} \coprod_{\mathfrak{c}_{L, 1}}^{\mathfrak{c}_{L, K}}-\mathbb{I}_{\mathscr{H}^{L}}\right]\right\|_{\mathcal{S}_{\mathscr{H}^{L}, \mathscr{H}}}^{2} \precsim \vartheta_{N}^{2} c_{L, K}^{-4}\left\|\boldsymbol{\pi}_{L}\right\|_{\mathcal{S}_{\mathscr{H}^{L}, \mathscr{H}}}^{2}=\mathrm{O}\left(c_{L, K}^{-4} \vartheta_{N}^{2}\right) .
$$

Since $L=\Xi(1, N), K=\Xi\left(1, \sqrt[3]{L^{-1} N}\right), c_{L, K}=\Omega\left(\sqrt{L^{3} N^{-1}}\right),\left(\boldsymbol{\pi}_{L},\left(\Phi_{L, i j}\right)_{i, j}\right)$ satisfies Assumption 3.4 for all $L \in \mathbb{N}$ for $\beta>0,(3.26)$ and $\vartheta_{N}=\mathrm{O}\left(c_{L, K}^{-2} K^{-\beta}\right)$, plugging (5.10)-(5.14) of $T_{1}-T_{5}$ into (5.9) implies

$$
\begin{aligned}
\left\|\hat{\boldsymbol{\pi}}_{L, K}-\boldsymbol{\pi}_{L}\right\|_{\mathcal{S}_{\mathscr{H}^{L}, \mathscr{H}}}^{2} & \precsim\left\|\hat{\boldsymbol{\pi}}_{L, K}-\coprod_{\mathscr{J}_{L, K}} \boldsymbol{\pi}_{L}\right\|_{\mathcal{S}_{\mathscr{H}^{L}, \mathscr{H}}}^{2}+\left\|\coprod_{\mathscr{J}_{L, K}^{c}} \boldsymbol{\pi}_{L}\right\|_{\mathcal{S}_{\mathscr{H}^{L}, \mathscr{H}}}^{2} \\
& =\mathrm{O}_{\mathbb{P}}\left(c_{L, K}^{-4} K L^{4} N^{-1}\right)+\mathrm{O}\left(L\left(\left.c_{L, K}^{-1} \sum_{l>L}\left\|\pi_{l}\right\|\right|_{\mathcal{L}_{\mathscr{H}}}\right)^{2}\right)+\mathrm{O}\left(K^{-2 \beta}\right) .
\end{aligned}
$$

Consequently, since we imposed $c_{L, K}^{-4} L^{4} N^{-1}=\mathrm{O}\left(K^{-(2 \beta+1)}\right)$ if $\sum_{l>L}\left\|\pi_{l}\right\|_{\mathcal{L}_{\mathscr{H}}}=\mathrm{O}\left(c_{L, K}^{-1} \sqrt{K L^{3} N^{-1}}\right)$ and $L^{-2} N\left(\sum_{l>L}\left\|\pi_{l}\right\|_{\mathcal{L}_{\mathscr{H}}}\right)^{4}=\mathrm{O}\left(K^{1-2 \beta}\right)$ if $c_{L, K}^{-1} \sqrt{K L^{3} N^{-1}}=\mathrm{O}\left(\sum_{l>L}\left\|\pi_{l}\right\|_{\mathcal{L}_{\mathscr{H}}}\right)$, where latter is because of $c_{L, K}=$ $\mathrm{O}\left(K^{-1} L\right)$ and $\sum_{l>L}\left\|\pi_{l}\right\|_{\mathcal{L}_{\mathscr{H}}}=\mathrm{o}(1)$ only possible if $K=\mathrm{o}\left(\sqrt[3]{L^{-1} N}\right),(3.43)$ is verified.

Proof of Theorem 3.5. The definition of $\hat{\boldsymbol{\beta}}_{[\mathfrak{q}]},(3.44)$ and the fact that $\left(\boldsymbol{\beta}_{[\mathfrak{q}]},\left(\Phi_{\mathfrak{p}, \mathfrak{q} ; i j}\right)_{i, j}\right)$ satisfies Assumption 3.4 for $\beta>0$, imply similarly as in the proof of Theorem 3.3:

$$
\begin{aligned}
& \left\|\hat{\boldsymbol{\beta}}_{[\mathfrak{q}]}-\boldsymbol{\beta}_{[\mathfrak{q}]}\right\|_{\mathcal{S}_{\mathscr{H}} \mathfrak{q}, \mathscr{H}}^{2} \precsim\left\|\hat{\boldsymbol{\beta}}_{[\mathfrak{q}]}-\coprod_{\mathscr{J}_{\mathfrak{p}, \mathfrak{q} ; M}} \boldsymbol{\beta}_{[\mathfrak{q}]}\right\|_{\mathcal{S}_{\mathscr{H}} \mathfrak{q}, \mathscr{H}}^{2}+\left\|\coprod_{\mathscr{J}_{\mathfrak{p}, \mathfrak{q} ; M}} \boldsymbol{\beta}_{[\mathfrak{q}]}\right\|_{\mathcal{S}_{\mathscr{H}} \mathfrak{q}, \mathscr{H}}^{2} \\
& \precsim\left\|\hat{\boldsymbol{\pi}}_{[\mathfrak{s , q}]}-\boldsymbol{\pi}_{[\mathfrak{s}, \mathfrak{q}]}\right\|_{\mathcal{S}_{\mathscr{H}} \mathfrak{q}, \mathscr{H}}^{2}\left\|\hat{\prod}_{[\mathfrak{s}, \mathfrak{q}]}^{\dagger} \coprod_{\hat{\mathfrak{g}}_{\mathfrak{s}, \mathfrak{q} ; 1}}^{\hat{\mathfrak{g}}_{\mathfrak{s}, \mathfrak{q} ; M}}\right\|_{\mathcal{L}_{\mathscr{H}} \mathfrak{q}}^{2}+\left\|\boldsymbol{\pi}_{[\mathfrak{s}, \mathfrak{q}]}\right\|_{\mathcal{S}_{\mathscr{H}} \mathfrak{q}, \mathscr{H}}^{2}\left\|\prod_{[\mathfrak{s}, \mathfrak{q}]}^{\dagger} \coprod_{\hat{\mathfrak{g}}_{\mathfrak{s}, \mathfrak{q} ; 1}}^{\hat{\mathfrak{g}}_{\mathfrak{s}, \mathfrak{q} ; M}}-\prod_{[\mathfrak{s}, \mathfrak{q}]}^{\dagger} \coprod_{\mathfrak{g}_{\mathfrak{s}, \mathfrak{q} ; 1}}^{\mathfrak{g}_{\mathfrak{s}, \mathfrak{q} ; M}}\right\|_{\mathcal{L}_{\mathscr{H}} \mathfrak{q}}^{2} \\
& +\left\|\boldsymbol{\pi}_{[\mathfrak{s}, \mathfrak{q}]} \prod_{[\mathfrak{s}, \mathfrak{q}]}^{\dagger} \coprod_{\mathfrak{g}_{\mathfrak{s}, \mathfrak{q} ; 1}}^{\mathfrak{g}_{\mathfrak{s}, \mathfrak{q} ; M}}-\coprod_{\mathscr{J}_{\mathfrak{p}, \mathfrak{q} ; M}} \boldsymbol{\beta}_{[\mathfrak{q}]}\right\|_{\mathcal{S}_{\mathscr{H} \mathfrak{q}, \mathscr{H}}}^{2}+\mathrm{O}\left(M^{-2 \beta}\right) \\
& \precsim \mathrm{O}_{\mathbb{P}}\left(K^{-2 \beta}\right)\left\|\hat{\prod}_{[\mathfrak{s}, \mathfrak{q}]}^{\dagger} \coprod_{\hat{\mathfrak{g}}_{\mathfrak{s}, \mathfrak{q} ; 1}}^{\hat{\mathfrak{g}}_{\mathfrak{s}, \mathfrak{q} ; M}}\right\|_{\mathcal{L}_{\mathscr{H}} \mathfrak{q}}^{2}+\left\|\hat{\prod}_{[\mathfrak{s}, \mathfrak{q}]}^{\dagger} \coprod_{\hat{\mathfrak{g}}_{\mathfrak{s}, \mathfrak{q} ; 1}}^{\hat{\mathfrak{g}}_{\mathfrak{s}, \mathfrak{q} ; M}}-\prod_{[\mathfrak{s}, \mathfrak{q}]}^{\dagger} \coprod_{\mathfrak{g}_{\mathfrak{s}, \mathfrak{q} ; 1}}^{\mathfrak{g}_{\mathfrak{s}, \mathfrak{q} ; M}}\right\|_{\mathcal{L}_{\mathscr{H} \mathfrak{q}}}^{2} \\
& +\left\|\coprod_{\mathscr{J}_{\mathfrak{p}, \mathfrak{q} ; M}^{c}} \boldsymbol{\beta}_{[\mathfrak{q}]} \prod_{[\mathfrak{s}, \mathfrak{q}]}^{\dagger} \coprod_{\mathfrak{g}_{\mathfrak{s}, \mathfrak{q} ; 1}}^{\mathfrak{g}_{\mathfrak{s}, \mathfrak{q} ; M}}\right\|_{\mathcal{S}_{\mathscr{H} \mathfrak{q}, \mathscr{H}}}^{2}+\left\|\coprod_{\mathscr{J}_{\mathfrak{p}, \mathfrak{q} ; M}} \boldsymbol{\beta}_{[\mathfrak{q}]}\left[\prod_{[\mathfrak{s}, \mathfrak{q}]}^{\ddagger} \coprod_{\mathfrak{g}_{\mathfrak{s}, \mathfrak{q} ; 1}}^{\mathfrak{g}_{\mathfrak{s}, \mathfrak{q} ; M}}-\mathbb{I}_{\mathscr{H} \mathfrak{q}}\right]\right\|_{\mathcal{S}_{\mathscr{H}} \mathfrak{q}, \mathscr{H}}^{2}+\mathrm{O}\left(M^{-2 \beta}\right) \\
& =: \mathrm{O}_{\mathbb{P}}\left(K^{-2 \beta}\right) \cdot T_{1}+T_{2}+T_{3}+T_{4}+\mathrm{O}\left(M^{-2 \beta}\right) \text {. }
\end{aligned}
$$


$\underline{\text { Term } T_{1}}$ : Since $\hat{\prod}_{[\mathfrak{s}, \mathfrak{q}]}^{\dagger}=\hat{\prod}_{[\mathfrak{s}, \mathfrak{q}]}^{*}\left(\hat{\prod}_{[\mathfrak{s}, \mathfrak{q}]} \hat{\prod}_{[\mathfrak{s}, \mathfrak{q}]}^{*}+\theta_{N} \mathbb{I}_{\mathscr{H} \mathfrak{q} \mathfrak{q}}\right)^{-1}$ and since $\left(\hat{\mathfrak{g}}_{\mathfrak{s}, \mathfrak{q} ; j}\right)_{j}$ is the eigenfunction sequence of $\hat{\prod}_{[\mathfrak{s}, \mathfrak{q}]} \hat{\prod}_{[\mathfrak{s}, \mathfrak{q}]}^{*}$, thus $\hat{\prod}_{[\mathfrak{s}, \mathfrak{q}]} \hat{\prod}_{[\mathfrak{s}, \mathfrak{q}]}^{*}$ and $\coprod_{\hat{\mathfrak{g}}_{\mathfrak{s}, q ; 1}}^{\hat{\mathfrak{s}}_{\mathfrak{s}, 1}}$ commute, yields similarly as in $T_{1}$ of Theorem 3.3 :

$$
\begin{aligned}
\left\|\hat{\prod}_{[\mathfrak{s , q}]}^{\dagger} \coprod_{\hat{\mathfrak{g}}_{\mathfrak{s}, q ; 1}}^{\hat{\mathfrak{g}}_{\mathfrak{s}, \mathfrak{q} ;} M}\right\|_{\mathcal{L}_{\mathscr{H}} \mathfrak{q}}^{2} & =\left\|\left(\hat{\prod}_{[\mathfrak{s , q}]} \hat{\prod}_{[\mathfrak{s}, \mathfrak{q}]}^{*}+\theta_{N} \mathbb{I}_{\mathscr{H} \mathcal{q}}\right)^{-2} \hat{\prod}_{[\mathfrak{s , q}]} \hat{\prod}_{[\mathfrak{s , q}]}^{*} \coprod_{\hat{\mathfrak{g}}_{\mathfrak{s}, q ; 1}}^{\hat{\mathfrak{g}}_{\mathfrak{s}, \mathfrak{q} ; M}}\right\|_{\mathcal{L}_{\mathscr{H}} \mathfrak{q}}=\sup _{j \leq M} \frac{\hat{g}_{\mathfrak{s}, \mathfrak{q} ; j}}{\left(\hat{g}_{\mathfrak{s}, \mathfrak{q} ; j}+\theta_{N}\right)^{2}} \\
& =\mathrm{O}_{\mathbb{P}}\left(g_{\mathfrak{s}, \mathfrak{q} ; M}^{-1}\right) .
\end{aligned}
$$

Term $T_{2}$ : Due to $\hat{\prod}_{[\mathfrak{s}, \mathfrak{q}]}^{\dagger}\left(\hat{\mathfrak{g}}_{\mathfrak{s}, \mathfrak{q} ; j}^{\prime \prime \prime}\right)=\left(\hat{g}_{\mathfrak{s}, \mathfrak{q} ; j}+\theta_{N}\right)^{-1} \hat{\prod}_{[\mathfrak{s}, \mathfrak{q}]}^{*}\left(\hat{\mathfrak{g}}_{\mathfrak{s}, \mathfrak{q} ; j}^{\prime \prime \prime}\right), \prod_{[\mathfrak{s , q}]}^{\dagger}\left(\mathfrak{g}_{\mathfrak{s}, \mathfrak{q} ; j}^{\prime \prime \prime}\right)=\left(g_{\mathfrak{s , q} ; j}+\theta_{N}\right)^{-1} \prod_{[\mathfrak{s}, \mathfrak{q}]}^{*}\left(\hat{\mathfrak{g}}_{\mathfrak{s}, \mathfrak{q} ; j}^{\prime \prime \prime}\right)$ and conversions as in $T_{2}$ in Theorem 3.3, we obtain

$$
\begin{aligned}
& \left\|\hat{\prod}_{[\mathfrak{s , q}]}^{\dagger} \coprod_{\hat{\mathfrak{g}}_{\mathfrak{s}, q ; 1}}^{\hat{\mathfrak{g}}_{\mathfrak{s}, q ;}}-\prod_{[\mathfrak{s , q}]}^{\dagger} \coprod_{\mathfrak{g}_{\mathfrak{s}, q ; 1}}^{\mathfrak{g}_{\mathfrak{s}, q ;} ;}\right\|_{\mathcal{L}_{\mathscr{H}} \mathfrak{q}}^{2} \\
& =\sup _{\|\boldsymbol{x}\|_{\mathscr{H} \mathfrak{q}} \leq 1}\left\|\sum_{j=1}^{M} \frac{\left\langle\boldsymbol{x}, \hat{\mathfrak{g}}_{\mathfrak{s} ; \mathfrak{q} ;}^{\prime \prime \prime}\right\rangle_{\mathscr{H} \mathfrak{q}}}{\hat{g}_{\mathfrak{s}, \mathfrak{q} ; j}+\theta_{N}} \hat{\prod}_{[\mathfrak{s , q}]}^{*}\left(\hat{\mathfrak{g}}_{\mathfrak{s}, \mathfrak{q} ; j}^{\prime \prime \prime}\right)-\frac{\left\langle\boldsymbol{x}, \mathfrak{g}_{\mathfrak{s}, \mathfrak{q} ; j}\right\rangle_{\mathscr{H} \mathfrak{q}}}{g_{\mathfrak{s}, \mathfrak{q} ; j}+\theta_{N}} \prod_{[\mathfrak{s , q}]}^{*}\left(\mathfrak{g}_{\mathfrak{s}, \mathfrak{q} ; j}\right)\right\|_{\mathscr{H} \mathfrak{q}}^{2}
\end{aligned}
$$

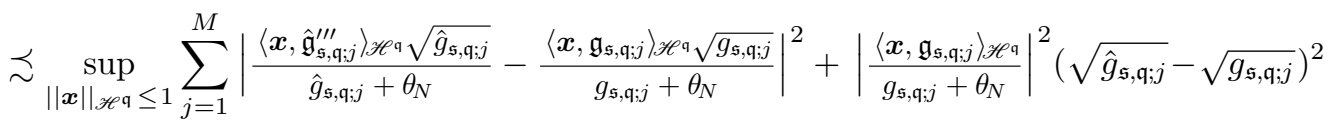

$$
\begin{aligned}
& +\sup _{j \leq M}\left|\frac{1}{g_{\mathfrak{s , q} ; j}+\theta_{N}}\right|^{2}\left(\left\|\hat{\prod}_{[\mathfrak{s , q}]}^{*}\right\|_{\mathcal{L}_{\mathscr{H} \mathfrak{q}}}^{2}\left\|\hat{\mathfrak{g}}_{\mathfrak{s , q} ; j ;}^{\prime \prime \prime}-\mathfrak{g}_{\mathfrak{s , q} ; j ;}\right\|_{\mathscr{H} \mathfrak{q}}^{2}+\left\|\hat{\prod}_{[\mathfrak{s , q}]}^{*}-\prod_{[\mathfrak{s , q}]}^{*}\right\|_{\mathcal{L}_{\mathscr{H} \mathfrak{q}}}^{2}\right)
\end{aligned}
$$

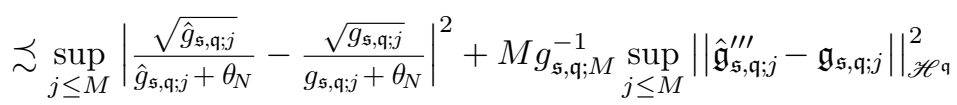

$$
\begin{aligned}
& +\sup _{j \leq M}\left(\frac{1}{g_{\mathfrak{s}, \mathfrak{q} ; j}+\theta_{N}}\right)^{2}\left(\left(\sqrt{\hat{g}_{\mathfrak{s}, \mathfrak{q} ; j}}-\sqrt{g_{\mathfrak{s}, \mathfrak{q} ; j}}\right)^{2}+\left\|\hat{\prod}_{[\mathfrak{s , q}]}^{*}\right\|_{\mathcal{L}_{\mathscr{H} \mathfrak{q}}}^{2}\left\|\hat{\mathfrak{g}}_{\mathfrak{s}, \mathfrak{q} ; j}^{\prime \prime \prime}-\mathfrak{g}_{\mathfrak{s , q} ; j}\right\|_{\mathscr{H}_{\mathfrak{q}}}^{2}+\left\|\hat{\prod}_{[\mathfrak{s , q}]}^{*}-\prod_{[\mathfrak{s , q}]}^{*}\right\|_{\mathcal{L}_{\mathscr{H} \mathfrak{q}}}^{2}\right) \\
& =\mathrm{O}_{\mathbb{P}}\left(g_{\mathfrak{s}, \mathfrak{q} ; M}^{-2} K^{-\beta}\right)+\mathrm{O}_{\mathbb{P}}\left(g_{\mathfrak{s}, \mathfrak{q} ; M}^{-3} K^{-2 \beta}\right)+\mathrm{O}_{\mathbb{P}}\left(g_{\mathfrak{s}, \mathfrak{q} ; M}^{-2} K^{-\beta}\right)+\mathrm{O}_{\mathbb{P}}\left(g_{\mathfrak{s}, \mathfrak{q} ; M}^{-4} K^{-2 \beta}\right)=\mathrm{O}_{\mathbb{P}}\left(g_{\mathfrak{s}, \mathfrak{q} ; M}^{-2} K^{-\beta}\right) .
\end{aligned}
$$

$\underline{\text { Term } T_{3}}$ : From the assumptions made and the idea of the derivation of $T_{3}$ in Theorem 3.3 follows

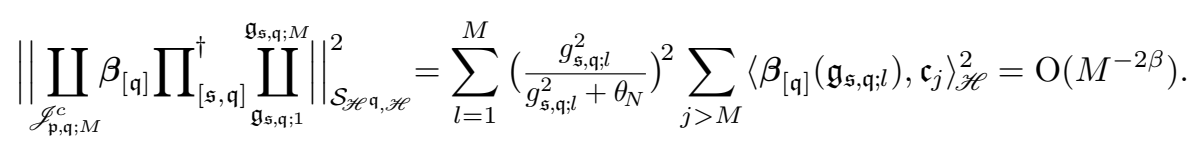

Term $T_{4}$ : Analogously as in $T_{4}$ in Theorem 3.3 , we obtain

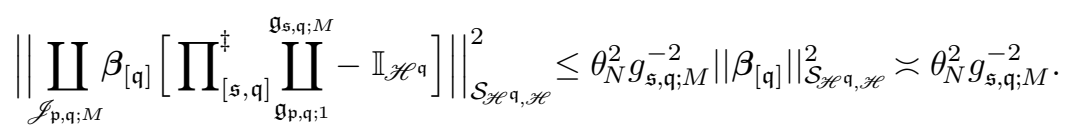

Replacing $T_{1}-T_{4}$ in (5.15) by (5.16)-(5.19) and considering $\theta_{N}=\mathrm{O}\left(K^{-\beta / 2}\right)$ and $g_{\mathfrak{s}, \mathfrak{q} ; M}^{-1} M^{\beta}=\mathrm{O}\left(K^{\beta}\right)$, yields

$$
\begin{aligned}
\left\|\hat{\boldsymbol{\beta}}_{[\mathfrak{q}]}-\boldsymbol{\beta}_{[\mathfrak{q}]}\right\|_{\mathcal{S}_{\mathscr{Y e q}, \mathscr{H}}}^{2} & =\mathrm{O}_{\mathbb{P}}\left(g_{\mathfrak{s , q} ; M}^{-1} K^{-\beta}\right)+\mathrm{O}_{\mathbb{P}}\left(g_{\mathfrak{s}, \mathfrak{q} ; M}^{-2} K^{-\beta}\right)+\mathrm{O}\left(M^{-2 \beta}\right)+\theta_{N}^{2} g_{\mathfrak{s , q} ; M}^{-2}+\mathrm{O}\left(M^{-2 \beta}\right) \\
& =\mathrm{O}_{\mathbb{P}}\left(M^{-2 \beta}\right)
\end{aligned}
$$

with what (3.50) is shown for all $j$ and (3.49) for $i=1, \ldots, \mathfrak{p}$ follows from (3.43) for all $i,(3.50)$ for all $j$ and

$$
\left\|\hat{\alpha}_{i}-\alpha_{i}\right\|_{\mathcal{S}_{\mathscr{H}}}^{2} \precsim\left\|\hat{\pi}_{i}-\pi_{i}\right\|_{\mathcal{S}_{\mathscr{H}}}^{2}+\sum_{j=1}^{i-1 \wedge \mathfrak{q}}\left\|\hat{\beta}_{j}\right\|_{\mathcal{S}_{\mathscr{H}}}^{2}\left\|\hat{\pi}_{i-j}-\pi_{i-j}\right\|_{\mathcal{S}_{\mathscr{H}}}^{2}+\left\|\hat{\beta}_{j}-\beta_{j}\right\|_{\mathcal{S}_{\mathscr{H}}}^{2}\left\|\pi_{i-j}\right\|_{\mathcal{S}_{\mathscr{H}}}^{2}
$$

\section{Acknowledgements}

This article contains the integral components of the doctoral thesis Über funktionale ARCH- und GARCHZeitreihen, Kühnert (2019) supervised and refereed by Alexander Meister (University of Rostock) and also refereed by Alexander Aue (UC Davis). I am thankful to Alexander Meister for various inspirational conversations as well as insightful advices and to Alexander Aue for valuable hints on this article and future work. I thank Dominik Liebl (University of Bonn) for helpful suggestions and also Leo Evans for proof-reading. 


\section{References}

[1] Andersen T. G., Davis R.A., Kreiss J.-P. \& Mikosch T.V. (2009). Handbook of Financial Time Series. Springer, Berlin.

[2] Aue A., Horváth L. \& Pellatt D.F. (2017). Functional generalized autoregressive conditional heteroskedasticity. Journal of Time Series Analysis 38, 3-21.

[3] Aue A. \& Klepsch J. (2017). Estimating functional time series by moving average model fitting. arXiv:1701.00770v1.

[4] Billingsley P. (1995), Probability and Measure. John Wiley \& Sons, Inc., New York.

[5] Bollerslev T. (1986). Generalized Autoregressive Conditional Heteroskedasticity. Journal of Econometrics 31, $307-327$.

[6] Bosq D. (2000), Linear Processes in Function Spaces. Springer, New York.

[7] Bosq D. \& Blanke P. (2007), Inference and Prediction in Large Dimensions. John Wiley \& Sons Ltd.

[8] Brockwell P. J. \& Davis, R. A. (2002). Introduction to Time Series and Forecasting. Springer, New York. (2 ${ }^{\text {nd }}$ Ed.).

[9] Cerovecki C., Francq C., Hörmann S. \& Zakoïan J.-M. (2019). Functional GARCH Models: The Quasi-likelihood Approach and Its Applications. Journal of Econometrics. https://doi.org/10.1016/j.jeconom.2019.01.006.

[10] Engle R.F. (1982). Autoregressive conditional heteroskedasticity with estimates of the variance of U.K.inflation. Econometrica 50 (4), 987-1008.

[11] Ferraty F. \& Vieu, P. (2006). Nonparametric Functional Data Analysis. Springer, New York.

[12] Francq C. \& Zakoïan, J.-M. (2010). GARCH Models: Structure, Statistical Inference and Financial Applications. John Wiley 8 Sons Ltd.

[13] Gohberg I., Goldberg S. \& Kaashoek M.A. (1990). Classes of Linear Operators, Vol.I Birkhäuser, Basel.

[14] Gouriéroux C. (1997). ARCH Models and Financial Applications. Springer, New York.

[15] Horváth L. \& Kokoszka P. (2012). Inference for Functional Data with Applications. Springer, New York.

[16] Hörmann S., Horváth L. \& Reeder R. (2013). A Functional Version of the ARCH Model. Econometric Theory 29(2), $267-288$.

[17] Hörmann S. \& Kokoszka P. (2010). Weakly dependent functional data. The Annals of Statistics 38, 1845-1884.

[18] Hsing T. \& Eubank R. (2015). Theoretical Foundations of Functional Data Analysis, with an Introduction to Linear Operators. Wiley, West Sussex, UK.

[19] Kaballo W. (2014). Aufbaukurs Funktionalanalysis und Operatortheorie. Springer Spektrum, Berlin.

[20] Kingman J.F.C. (1974). Subadditive Ergodic Theory. Annals of Probability 6, 883-909.

[21] Klepsch J., Klüppelberg C. \& Wei T. (2017). Prediction of functional ARMA processes with an application to traffic data. Econometris and Statistics 1, 128-149.

[22] Klepsch J. \& Klüppelberg C. (2017). An Innovations Algorithm for the prediction of functional linear processes. Journal of Multivariate Analysis 155, 252-271.

[23] Kokoszka P. \& Reimherr M. (2013). Determing the order of the functional autoregressive model. Journal of Time Series Analysis 34, 116-129.

[24] Kokoszka P., Rice G. \& Shang H.L. (2017). Inference for the autocovariance of a functional time series under conditional heteroscedasticity. Journal of Multivariate Analysis 162, 32-50.

[25] Kühnert S. (2019). Über funktionale ARCH- und GARCH-Zeitreihen. Doctoral Thesis, University of Rostock.

[26] Ledoux M. \& Talagrand M. (1991). Probability in Banach Spaces. Springer, Berlin.

[27] Ramsay J.O. \& Silverman B.W. (1997). Functional Data Analysis. Springer, New York.

[28] Rice G., Wirjanto, T. \& Zhao Y. (2019). Tests for conditional heteroscedasticity with functional data and goodness-of-fit tests for FGARCH models. https://mpra.ub.uni-muenchen.de/93048/

[29] Ruiz-Medina M.D. \& Álvarez-Liébana J. (2019). Strongly consistent autoregressive predictors in abstract Banach spaces. Journal of Multivariate Analysis 170, 186-201.

[30] Spangenberg F. (2013). Strictly stationary solutions of ARMA equations in Banach spaces. Journal of Multivariate Analysis 121, 127-138.

[31] Stout W.F. (1974). Almost Sure Convergence. Academic Press, New York.

[32] Tichonow A. N. \& Arsenin V.Y. (1977). Solutions of Ill-Posed Problems. Winston and Sons, Washington DC.

[33] Turbillon C., Marion J.-M. \& Pumo B. (2007). Estimation of the moving-average operator in a Hilbert space. Recent advances in stochastic modeling and data analysis. World Sci. Publ., Hackensack, NJ, 597-604.

[34] Werner D. (2011). Funktionalanalysis. Springer, Berlin ( $7^{\text {th }}$ Ed.). 